\title{
Temporal derivative computation in the dorsal raphe network revealed by an experimentally-driven augmented integrate-and-fire modeling framework
}

\author{
Emerson F. Harkin ${ }^{1}$, Alexandre Payeur ${ }^{1,2,3}$, Michael B. Lynn ${ }^{1}$, \\ Jean-François Boucher ${ }^{1}$, Léa Caya-Bissonnette ${ }^{1}$, Dominic Cyr ${ }^{1}$, Chloe Stewart ${ }^{1,4}$, \\ André Longtin $^{1,2}$, Richard Naud ${ }^{* 1,2}$, Jean-Claude Béique*11
}

1: uOttawa Brain and Mind Institute, Centre for Neural Dynamics, Department of Cellular and Molecular Medicine, University of Ottawa, 451 Smyth Rd, Ottawa, ON, K1H 8M5, Canada

2: Department of Physics, University of Ottawa, 150 Louis Pasteur Pvt, Ottawa, ON, K1N $6 \mathrm{~N} 5$, Canada

3: Present address: Mila, Université de Montréal, Montréal, QC, Canada

4: Present address: Department of Biology, McGill University, 3649 Promenade Sir-William-Osler, Montréal, QC, H3A 1W9, Canada

*: co-corresponding authors: rnaud@uottawa.ca, ibeique@uottawa.ca 


\section{Abstract}

By means of an expansive innervation, the relatively few phylogenetically-old serotonin (5-HT) neurons of the dorsal raphe nucleus (DRN) are positioned to enact coordinated modulation of circuits distributed across the entire brain in order to adaptively regulate behavior. In turn, the activity of the DRN is driven by a broad set of excitatory inputs, yet the resulting network computations that naturally emerge from the excitability and connectivity features of the various cellular elements of the DRN are still unknown. To gain insight into these computations, we developed a flexible experimental and computational framework based on a combination of automatic characterization and network simulations of augmented generalized integrate-and-fire (aGIF) single-cell models. This approach enabled the examination of causal relationships between specific excitability features and identified population computations. We found that feedforward inhibition of 5-HT neurons by heterogeneous DRN somatostatin (SOM) neurons implemented divisive inhibition, while endocannabinoid-mediated modulation of excitatory drive to the DRN increased the gain of 5-HT output. The most striking computation that arose from this work was the ability of 5-HT output to linearly encode the derivative of the excitatory inputs to the DRN. This network computation primarily emerged from the prominent adaptation mechanisms found in 5-HT neurons, including a previously undescribed dynamic threshold. This novel computation in the DRN provides a potential mechanism underlying some of the functions recently ascribed to 5 -HT in the context of reinforcement learning. 


\section{Introduction}

The forebrain-projecting serotonin (5-HT) neurons of the DRN play a key role in regulating behaviour in dynamic environments, but the precise nature of this role is still not well understood (Young et al. 1985; Delgado et al. 1994; Warden et al. 2012; Dayan and Huys, 2015). DRN serotonin neurons have been proposed to modulate a wide range of cognitive processes, such as encouraging patience for future rewards (Miyazaki et al. 2014; Fonseca et al. 2015), signalling the beneficialness of current actions or states (Luo et al. 2016), complementing reinforcement signals of dopamine (Daw et al. 2002, Maier et al. 2005, Nakamura et al. 2008, Ranade et al. 2009, Tops et al. 2009, Cools et al., 2011, Li et al. 2016), and, partially as a corollary, regulating both learning (Soubrié 1986, Deakin 1991, Daw et al. 2002, Dayan and Huys 2009, Matias et al., 2017) and mood (Savitz et al., 2009, Fava and Kendler 2000, Donaldson et al., 2013). While the remarkable diversity of roles attributed to this single neurotransmitter has historically been perplexing, recent findings are beginning to provide insight (see Okaty et al., 2019 for review). For example, the unsuspected organization of 5-HT neurons into anatomical sub-modules that differentially regulate behaviour (Abrams et al. 2004; Lowry et al. 2005; Commons et al. 2015; Muzerelle et al. 2016; Ren et al. 2018), or the observation that 5-HT neurons can encode distinct salient features of the environment over different time scales (Trulson and Jacobs, 1979;

Schweimer and Ungless 2010; Ranade et al. 2009; Cohen et al., 2015, Zhong et al., 2017) are compelling mechanisms that may contribute to the multiplicity of 5-HT's actions. These anatomical and dynamical perspectives on 5-HT diversity need not be mutually exclusive. A clearer understanding of the biophysical mechanisms that contribute to the coding features of raphe neurons over multiple timescales has the potential to substantially increase our understanding of how 5 -HT regulates behavior.

The spiking statistics of 5-HT neurons necessarily shape and constrain their computational role. For instance, the slow firing rate $(\sim 5 \mathrm{~Hz})$ of 5 -HT neurons, in large part attributable to a large after-hyperpolarization potential (AHP) (Aghajanian \& Vandermaelen 1982; Vandermaelen \& Aghajanian, 1983), may appear to preclude signalling on faster time scales. However, fast signalling despite slow firing can arise naturally in ensemble-rate codes (Knight 1972; Gerstner 2000). Consistent with this idea, the in vivo population activity of 5-HT neurons has been observed to track impending rewards over second to sub-second timescales (Zhong et al., 2017), and the trial-averaged ensemble rates of individual 5-HT neurons can track environmental changes over the millisecond time scale (Ranade et al. 2009; Cohen et al. 2015). In addition, the fact that 5-HT receptor subtypes can regulate the excitability of target neurons over different time scales, including ionotropic 5-HT3 receptors with millisecond gating kinetics (Béïque et al. 2004; 2007; Andrade 2011; Varga et al., 2009) at the very least suggests that the 5-HT system is capable of fast information transmission, an observation mirrored by the fast dynamics of neurons which project to the DRN (Amo et al., 2014; Matsumoto and Hikosaka, 2007). If fast and slow signaling by the DRN is manifest, it is less clear which cellular mechanisms regulate the interplay between these timescales, nor which input features are represented on which timescales.

Computational modeling is a standard approach to link levels of description and is thus well suited to delineate how network-level function emerges from excitability features identified 
at the single-cell level. In spite of their conceptual utility, the most detailed single cell models, including those of DRN neurons (Tuckwell and Penington, 2014; Wong-Lin et al., 2017), do not lend themselves with ease to bottom-up modeling efforts because of the substantial technical difficulty of obtaining sufficiently accurate values for a large number of interacting model parameters (Prinz et al. 2004; Gerstner and Naud, 2009). Mathematically simpler generalized integrate-and-fire (GIF) models provide a strong foundation for network modelling because their small number of parameters can be estimated with a high degree of precision (Mensi et al. 2012, Pozzorini et al. 2013, Teeter et al. 2018). This precision comes at a price, however: the process of distilling the effects of many biophysical mechanisms into a small number of model parameters makes it difficult to study a specific mechanism (e.g., a subthreshold ion channel) in isolation. A hybrid approach based on a reductionistic GIF model augmented with a limited set of biophysical mechanisms could leverage the precision of GIFs while allowing the ability to link specific biophysical mechanisms with higher-order network function.

In this study, we developed and validated for DRN neurons a hybrid modeling approach that lies between reductionist GIF and biophysical Hodgkin-Huxley-type models to capture excitability features of individual neurons for accurate simulations of population dynamics and, by extension, network computation inference. To this end, we carried out cellular electrophysiological recordings from genetically-identified DRN 5-HT and SOM neurons to 1) extract and validate, from sets of noisy inputs, parameters for the automatic development of accurate GIF models, and; 2) experimentally define complementary biophysical mechanisms to be grafted onto the GIF models to iteratively improve their prediction accuracy (augmented GIFs). This approach recapitulated and extended past findings on DRN neurons by showing that the best-performing models of 5-HT neurons featured slow membrane time constants, an A-type potassium current, and strong adaptation mechanisms, including a previously unreported dynamic spike threshold. Network simulations of optimized GIF models of both 5-HT and GABAergic SOM neurons organized in a feed-forward inhibitory circuit revealed that 5-HT neuron populations encode the derivative of their inputs. Our overall approach further allowed us to trace back specific features of these population responses (e.g., gain) to defined excitability features of DRN neurons. 


\section{Materials and methods}

\section{Experimental methods}

\section{Animals}

Experiments were performed on male and female C57/Bl6 mice aged 4-8 weeks. SERT-Cre::Rosa-TdTomato and SOM-Cre::Rosa-TdTomato transgenic lines were used to fluorescently label DRN 5-HT and SOM GABA neurons, respectively. Animals were group-housed and kept on a 12:12-h light/dark cycle with access to food and water ad libitum. All experiments were carried out in accordance with procedures approved by the University of Ottawa Animal Care and Veterinary Services.

\section{Slice preparation}

Animals were deeply anaesthetized using isofluorane (Baxter Corporation) before being euthanized by decapitation. The brain was quickly removed from the skull and submerged into ice-cold dissection buffer containing the following: $119.0 \mathrm{mM}$ choline chloride, $2.5 \mathrm{mM}$ $\mathrm{KCl}, 4.3 \mathrm{mM} \mathrm{MgSO}_{4}, 1.0 \mathrm{mM} \mathrm{CaCl}_{2}, 1.0 \mathrm{mM} \mathrm{NaH} \mathrm{PO}_{4}, 1.3 \mathrm{mM}$ sodium ascorbate, $11.0 \mathrm{mM}$ glucose, $26.2 \mathrm{mM} \mathrm{NaHCO}$; saturated with $95 \%$ 02/5 \% CO2. A Leica VT1000S vibratome was used to cut $300 \mu \mathrm{m}$ coronal sections of midbrain containing the DRN or of the cortex containing the mPFC in the same ice-cold choline dissection buffer. After cutting, slices were placed in a recovery chamber filled with artificial cerebrospinal fluid (ACSF) containing the following: $119.0 \mathrm{mM} \mathrm{NaCl}, 2.5 \mathrm{mM} \mathrm{KCl}, 1.3 \mathrm{mM} \mathrm{MgSO}_{4}, 2.5 \mathrm{mM} \mathrm{CaCl}_{2}, 1.0 \mathrm{mM} \mathrm{NaH}_{2} \mathrm{PO}_{4}, 11.0$ $\mathrm{mM}$ glucose, $26.2 \mathrm{mM} \mathrm{NaHCO}_{3} ; \sim 298 \mathrm{mOsm}$, maintained at $37^{\circ} \mathrm{C}$, and continuously bubbled with $95 \% 02 / 5 \%$ CO2. The recovery chamber was allowed to equilibrate to room temperature for $1 \mathrm{~h}$ before beginning experiments.

\section{In vitro whole-cell electrophysiological recording}

Neurons were visualized using an upright microscope (Olympus BX51WI) equipped with differential interference contrast and a $40 \times, 0.8 \mathrm{NA}$ water-immersion objective. Whole-cell recordings were obtained from fluorescently-labelled DRN 5-HT and SOM neurons and unlabeled mPFC L5 pyramidal neurons using glass electrodes (Sutter Instruments; tip resistance 4 to $6 \mathrm{MOhm}$ ). For most experiments, the following potassium gluconate-based internal solution was used: $135 \mathrm{mM}$ potassium gluconate, $6.98 \mathrm{mM} \mathrm{KCl}, 10 \mathrm{mM}$ HEPES, 4 mM Mg ATP, $0.40 \mathrm{mM}$ GTP, $10 \mathrm{mM}$ Na phosphocreatine; adjusted to pH 7.25 with KOH, 280 to $290 \mathrm{mOsm}$. A subset of experiments (GABA synaptic physiology) were carried out using a cesium-based internal solution (120 mM CsMeSO 3 , 10 mM EGTA, 5 mM TEA Cl, 1 mM CaCl2, 10 mM Na HEPES, 4 mM Mg ATP, 2 mM GTP, 2 mM QX-314, and 10 mM Na phosphocreatine; adjusted to $\mathrm{pH} 7.25$ with $\mathrm{CsOH}, 280$ to $290 \mathrm{mOsm}$ ) and in the presence of bath-applied 100 $\mu \mathrm{M}(2 \mathrm{R})$-amino-5-phosphonovaleric acid (APV) and $5 \mu \mathrm{M}$

2,3-Dioxo-6-nitro-1,2,3,4-tetrahydrobenzo[f] quinoxaline-7-sulfonamide (NBQX). For voltage clamp experiments, whole-cell capacitance compensation was applied manually following break-in, and leak current subtraction was performed post hoc using membrane leak conductance estimated based on a $-5 \mathrm{mV}$ pulse at the start of each sweep. All 
experiments were carried out at room temperature. Neurons with access resistance $>30$ MOhm (or > 10 MOhm for synaptic physiology experiments) or unstable holding current at $-70 \mathrm{mV}$ were excluded from analysis. Recordings were collected with an Axon MultiClamp $700 \mathrm{~B}$ amplifier and the analog signals were filtered at $2 \mathrm{kHz}$ and digitized at $10 \mathrm{kHz}$ using an Axon Digidata 1550 digitizer.

\section{Models}

\section{GIF and related models}

The generalized integrate-and-fire (GIF) and Na-inactivation GIF (iGIF) models have been described previously in detail (Mensi et al., 2012, Pozzorini et al., 2015, Mensi et al., 2016). Briefly, the GIF and iGIF are composed of a subthreshold component which integrates input currents into voltage and a stochastic spiking rule which transforms subthreshold voltage into a series of spikes. The subthreshold dynamics of the GIF and iGIF are given by

$$
C \frac{d V}{d t}=-g_{l}\left(V(t)-E_{l}\right)-\sum_{\hat{t}_{i}<t} \eta\left(t-\hat{t}_{i}\right)+I_{\mathrm{inj}}(t)
$$

where $\left\{t_{i}^{\wedge}\right\}$ is the set of spike times and $\eta(t)=\sum_{j} \beta_{j}^{(\eta)} e^{\frac{-t}{\tau_{j}^{(\eta)}}}$ is the spike-triggered adaptation current. Here the $\beta_{j}^{(\eta)}$ are coefficients estimated from the data and the $\tau_{j}^{(\eta)}$ are fixed hyperparameters; see appendix for details. The GIF emits spikes according to an inhomogeneous Poisson process with intensity $\lambda(t)$, given by

$$
\lambda(t)=\lambda_{0} \exp \left[\frac{V(t)-V_{T}^{*}-\sum_{\hat{t}_{i}<t} \gamma\left(t-\hat{t}_{i}\right)}{\Delta V}\right]
$$

where $V_{T}^{*}$ is the stationary threshold, $\gamma(t)=\sum_{j} \beta_{j}^{(\gamma)} e^{-t / \tau_{j}^{(\gamma)}}$ is the spike-triggered threshold movement (where the $\beta_{j}^{(\gamma)}$ are coefficients estimated from the data and the $\tau_{j}^{(\gamma)}$ are fixed; see appendix), $\Delta V$ is the threshold sharpness ( $\mathrm{mV}$; larger values increase the stochasticity of spiking), and $\lambda_{0}=1 \mathrm{~Hz}$ is a constant such that $\lambda(t)$ is in units of $\mathrm{Hz}$. In the iGIF, an additional variable $\theta(t)$ is added to the numerator of the exponentiated term in Eq. 2 to account for voltage-dependent changes in threshold

$$
\lambda(t)=\lambda_{0} \exp \left[\frac{V(t)-V_{T}^{*}-\sum_{\hat{t}_{i}<t} \gamma\left(t-\hat{t}_{i}\right)+\theta(t)}{\Delta V}\right]
$$




$$
\frac{d \theta}{d t}=\frac{\theta_{\infty}(V)-\theta}{\tau^{(\theta)}}
$$

where the equilibrium voltage-dependent change in spike threshold

$\theta_{\infty}(V)=\sum_{j} \beta_{j}{ }^{(\theta)} \operatorname{rect}\left[V ; A_{j} A_{j+1}\right]$ is a piecewise constant function of voltage $\left(\right.$ each $\beta_{j}{ }^{(\theta)}$ defines the value of $\theta_{\infty}(V)$ from $A_{j}$ to $A_{j+1}$; see Mensi et al., 2016 for details on the iGIF). Our aGIF is identical to the GIF except that two Hodgkin-Huxley currents which together capture the voltage-gated potassium currents found in 5-HT neurons (see "Potassium current," below) are added to the subthreshold dynamics given in Eq. 1, yielding

$$
C \frac{d V}{d t}=-g_{l}\left(V(t)-E_{l}\right)-I_{A}(t)-I_{K}(t)-\sum_{t_{i}<t} \eta\left(t-t^{\wedge}{ }_{i}\right)+I_{i n j}(t)
$$

as the definition of the subthreshold dynamics of the aGIF.

The procedures for fitting the GIF and iGIF to electrophysiological data have also been described previously in detail (Mensi et al. 2012, Pozzorini et al. 2015, Mensi et al. 2016). Briefly, parameter estimation for both models occurs in two stages: first, the subthreshold parameters are estimated by regression, and second, the threshold parameters are estimated by maximizing the likelihood of the observed spiketrain as a function of the threshold parameters. The fitting procedure for the aGIF is very similar to that of the GIF, with adjustments to the subthreshold fitting procedure to accommodate the extra terms in Eq. 3 (see appendix for details).

\section{LIF neuron with an inactivating potassium current}

Our toy model of a neuron with an inactivating potassium current is based on a LIF augmented with $I_{A}(t)$ (see "Potassium current" below):

$$
C \frac{d V}{d t}=-g_{l}\left(V(t)-E_{l}\right)-I_{A}(t)+I_{i n j}(t),
$$

where $g_{l}$ and $E_{l}$ are the leak conductance and reversal, respectively, and $I_{i n j}(t)$ is the external input to the model. To reduce the number of free parameters, the model we used is non-dimensionalized with respect to the membrane time constant $\tau_{\text {mem }}=R_{\text {mem }} C$ and leak conductance $g_{l}$, yielding

$$
\frac{d V}{d t}=E_{l}-V(t)-\bar{g}_{A}^{\prime} m_{\infty} h\left(V(t)-E_{K}\right)+V_{i n j}(t)
$$


where $t$ is in units of the membrane time constant, $\bar{g}_{A}^{\prime}=\bar{g}_{A}{ }_{A} / g_{l}$ is the effective maximum conductance associated with $I_{A}$, and $V_{i n j}(t)=I_{i n j}(t) / g_{l}$ is the effective external input. The gating variables $m_{\infty}$ and $h$ are described below in "Potassium current."

\section{Potassium current}

The voltage-gated potassium currents in 5-HT neurons were modeled in terms of an inactivating current and a non-inactivating current we refer to as $I_{A}$ and $I_{K}$, respectively. These were defined as follows

$$
\begin{gathered}
I_{A}=\bar{g}{ }_{A} m_{\infty}(V) h(t)\left(V(t)-E_{K}\right) \\
I_{K}=\bar{g}{ }_{K} n_{\infty}(V)\left(V(t)-E_{K}\right)
\end{gathered}
$$

where $\bar{g}_{i}$ is the maximal conductance; $m$ and $h$ are the activation and inactivation gates of $I_{A}$, respectively; $n$ is the activation gate of $I_{K}$; and $\mathrm{E}_{\mathrm{K}}=-101 \mathrm{mV}$ is the reversal potential of potassium. The equilibrium state of each gate $x \in\{m, h, n\}$ is a sigmoid function of voltage

$$
x_{\infty}(V)=\frac{A_{x}}{1+e^{-k_{x}\left(V-V_{x}^{*}\right)}},
$$

where $V_{x}{ }^{*}$ is the half-activation voltage $(\mathrm{mV}), k_{x}$ is the slope $\left(\mathrm{mV}^{-1}\right)$, and $A_{x}$ is a scaling factor.

To keep the number of parameters in our current model to a minimum, we assumed that the $m$ and $n$ gates have instantaneous kinetics [allowing their corresponding equilibrium gating functions $m_{\infty}$ and $n_{\infty}$ to be used directly in Eq. 4], and that the $h$ gate activates and deactivates with a single time constant $\tau_{h}$ (ms) that does not depend on voltage. The time dynamics of the $h$ gate are therefore given by

$$
\frac{d h}{d t}=\frac{h_{\infty}-h}{\tau_{h}} .
$$

\section{Quantification of single neuron model performance}

$R^{2}$ was calculated based on the training set $\frac{d V}{d t}$ predicted by the subthreshold component of a given GIF model (Eqs. 1 and 3, where the spike times $\hat{t}$ were constrained to match the data), excluding a small window around each spike (from $1.5 \mathrm{~ms}$ before to $6.5 \mathrm{~ms}$ after in 5-HT neurons, and from $1.5 \mathrm{~ms}$ before to $4.0 \mathrm{~ms}$ after in SOM and mPFC neurons). $M_{d}{ }^{*}$ was calculated based on validation set data as previously described by Naud et al. (2011). This metric is defined as 


$$
M_{d}^{*}=\frac{2 n_{d m}}{n_{d d}{ }^{*}+n_{m m}},
$$

where $n_{d m}$ is the number of model-predicted spikes that occur within $8 \mathrm{~ms}$ of a spike in the validation data, and $n_{d d}{ }^{*}$ and $n_{m m}$ are the corresponding numbers of coincident spikes across sweeps in the validation data and model predictions (where $n_{d d}{ }^{*}$ is corrected for small sample bias). $M_{d}^{*}$ can be interpreted as the fraction of model-predicted spikes that occur within $8 \mathrm{~ms}$ of a spike emitted by a real neuron, corrected such that the chance level is 0 and perfect agreement between predicted and observed spikes is 1 .

\section{Population models}

DRN network models were constructed by connecting a population of 400 SOM neuron models to a population of 6005 -HT neuron models in a feed-forward arrangement. Population models were bootstrapped by sampling with replacement from a bank of experimentally-constrained GIF models. SOM neuron models were randomly connected to 5-HT neuron models with a connection probability of $2 \%$, such that the expected number of GABAergic synapses on each 5-HT neuron model was 8 . We used a conductance-based model of GABAergic synapses with a fixed reversal potential of $-76.7 \mathrm{mV}$, conductance of 0.3 $\mathrm{nS}$, and biexponential kinetics with $\tau_{\text {rise }}=1.44 \mathrm{~ms}, \tau_{\text {decay }}=26.0 \mathrm{~ms}$, and a propagation delay of $2.0 \mathrm{~ms}$.

Simulated 5-HT populations with increased or decreased $I_{A}$ were generated by setting $\bar{g}_{K 1}$ in all single neuron models to $0 \mathrm{nS}$ or $10 \mathrm{nS}$, respectively. DRN network models with homogenized SOM neuron populations were created by setting all SOM neuron model parameters to their respective median values from the bank of experimentally-constrained single neuron models. Population models in which the adaptation mechanisms of 5-HT and SOM neuron models were swapped were generated by randomly sampling a GIF model of the opposite cell type and substituting in its adaptation filter coefficients $\beta_{j}{ }^{(\gamma)}$ and $\beta_{j}^{(\eta)}$.

This procedure is summarized in the following pseudocode:

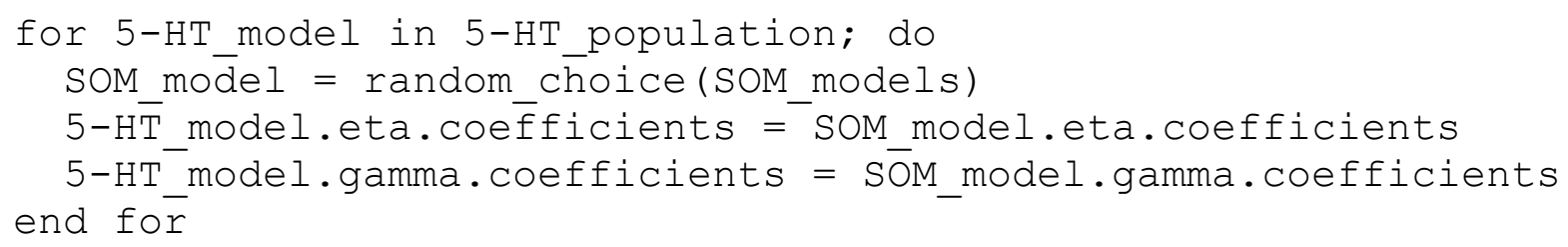

\section{Numerical methods}

Simulations were implemented in Python and $\mathrm{C}++$ using custom-written extensions of the GIF Fitting Toolbox (Pozzorini et al., 2015). Numerical integration was performed using the Euler method with a time step of $0.1 \mathrm{~ms}$ for the GIF and related models (to match the 
sampling rate of electrophysiological recordings) and $0.001 \tau_{\text {mem }}$ for the toy model of a neuron with $I_{A}$.

\section{Statistics}

Statistical analysis was carried out using the SciPy and statannot (https://github.com/webermarcolivier/statannot) Python packages. Non-parametric tests were used for all two-sample comparisons (Mann-Whitney U test for unpaired samples and Wilcoxon signed-rank test for paired samples). Where applicable, $p$-values were adjusted for multiple comparisons using the Bonferroni correction. ' $*$ ' ' '**', '***', and '****' are used in figures to denote statistical significance at the $p \leq 0.05,0.01,0.001$, and 0.0001 levels, respectively, and ' $o$ ' is used to indicate a trend towards significance (defined as $0.05<p \leq 0.1$ ). Exact $p$-values are reported in the main text, and summary statistics are presented as mean \pm standard-deviation.

\section{Results}

\section{Salient electrophysiological features of DRN neurons}

Our main goal was to develop an experimentally-grounded model of the DRN to better understand its computational properties. As a first step towards this goal, we carried out experiments to constrain a set of single-neuron models of the two main cell types found in the DRN: 5-HT and SOM GABA neurons. We performed whole-cell electrophysiological recordings from genetically-identified 5-HT (Fig 1A1; SERT-Cre::Rosa-TdTomato mice) and SOM (Fig 1A2; Table 1; SOM-Cre::Rosa-TdTomato mice) neurons in slices. In keeping with previous descriptions (e.g., Vandermaelen \& Aghajanian, 1983, Calizo et al. 2011), in the majority of the 5-HT neurons recorded in our dataset, current steps induced strongly adapting action potential firing accompanied by large afterhyperpolarization potentials (AHP), and a characteristic kink in the voltage trace leading up to the first spike (Fig. 1B). Qualitatively distinct firing patterns of 5-HT neurons were, however, occasionally observed (Fig. 1S1). Recordings from SOM neurons revealed spiking patterns that were more heterogeneous (Fig. 1S2). Comparing the relationship between the injected currents and firing frequencies between these populations, we found that SOM neurons were generally more sensitive to changes in input current (gain) than 5-HT neurons and responded to weaker inputs (Fig. 1C3). The gain showed greater variability in SOM neurons than in 5-HT neurons (Brown-Forsythe equality of variance test $p=0.001$ on $N=175$-HT and $N=7$ SOM neurons). In line with this observation, SOM neurons also consistently exhibited a wider range of firing frequencies for a given input $(e . g$, for a $50 \mathrm{pA}$ input 5-HT neurons fired at $2.81 \pm 2.22 \mathrm{~Hz}$ vs $8.16 \pm 5.70 \mathrm{~Hz}$; Brown-Forsythe test $p=0.005$ in $N=175$-HT neurons and $N=14$ SOM cells). Together, these observations outlined three salient cellular-level features of DRN neurons, namely the strong AHP and voltage kink of 5-HT neurons as well as noticeable heterogeneous excitability of SOM neurons. 
Table 1 Membrane parameters of DRN neurons. Parameters obtained from recordings from PFC L5 pyramidal neurons used to fit GIF models as a point of comparison are also shown. Data are presented as mean \pm SD. Distributions are shown in Fig. $1 S 3$.

\begin{tabular}{ccccc} 
Cell type & $R(\mathrm{G} \Omega)$ & $C(\mathrm{pF})$ & $\tau(\mathrm{ms})$ & $N$ \\
\hline 5-HT & $1.14 \pm 0.62$ & $65.7 \pm 17.1$ & $72.2 \pm 36.1$ & 112 \\
SOM & $1.07 \pm 0.58$ & $43.5 \pm 15.5$ & $42.2 \pm 19.8$ & 28 \\
mPFC & $0.188 \pm 0.130$ & $160.6 \pm 48.2$ & $27.4 \pm 13.2$ & 25
\end{tabular}

The characteristic kink in the voltage leading up to the first spike in 5-HT neurons in principle may be caused by near-threshold activation of voltage-gated potassium channels (VGKCs; Connor and Stevens 1971; Connor et al. 1977; Drion et al. 2015). We therefore examined whole-cell currents evoked by voltage steps (from $-90 \mathrm{mV}$ to $-20 \mathrm{mV}$ ) in both 5-HT and SOM neurons to look for evidence of such a VGKC. In 5-HT cells, these experiments revealed a large (peak amplitude $928 \pm 249 \mathrm{pA}$, leak-subtracted), partly-inactivating (steady-state amplitude $142 \pm 45 \mathrm{pA}$, leak-subtracted) outward current (Fig. 1C1) that was sensitive to potassium channel blockers (Fig. 1S4). This current activated rapidly (peak latency $7.46 \pm 0.21 \mathrm{~ms}$ ) and inactivated over tens of milliseconds (inactivation time constant $\tau_{h}=42.9 \pm 9.4 \mathrm{~ms}$ ). The gating and kinetic profile (Table 2 ) of the inactivating component of this conductance in 5-HT neurons are broadly similar to those expected of the A-type potassium currents $\left(I_{A}\right)$ characterized in great detail in several other cell types (e.g., Storm 1989). Because these parameters are sufficient to construct a model of this conductance (see below), we have not attempted to determine its molecular identity further. For the sake of simplicity, we refer to the inactivating component herein as $I_{A}$ (in keeping with the previous literature; see Aghajanian, 1985; Tuckwell and Penington 2014) and the steady-state component as $I_{K}$. Thus, an $I_{A}$-like inactivating VGKC is a consistent feature of DRN 5-HT neurons.

The same voltage-clamp protocol applied to SOM neurons, in contrast, triggered a mixture of outward and inward currents that varied widely from cell to cell (Fig. 1D2). A significant proportion of SOM neurons did not express a transient outward current at all $(27.3 \%$, Fig. $1 \mathrm{E})$, while those that did had currents that were significantly smaller $(p=0.003)$, activated more slowly ( $p=0.003)$, and exhibited much more heterogeneous kinetic profiles than those found in 5-HT neurons (Fig. 1E3). Together, these results show that the expression of this subthreshold voltage-gated current is substantially more variable in SOM neurons than in 5-HT neurons, in line with the distinctive heterogeneity of excitability features observed in this DRN cell type (Fig. 1C2 and 1S2). 
bioRxiv preprint doi: https://doi.org/10.1101/2021.06.25.449907; this version posted June 27, 2021. The copyright holder for this preprint (which was not certified by peer review) is the author/funder, who has granted bioRxiv a license to display the preprint in perpetuity. It is made available under aCC-BY-NC-ND 4.0 International license.

Table 2 5-HT $I_{A}$ current gating parameters. Gating curves shown in Fig. 2S1B were fitted with the scaled Boltzmann function $g_{\infty}(V) / g_{\infty}\left(V_{\text {reference }}\right)=x_{\infty}=A_{x} /\left(1+\exp \left[-k_{x}\left(V-V_{x}^{*}\right)\right]\right)$. Values are based on experiments from $N=13$ cells.

\begin{tabular}{ccccc} 
Gate & $V_{\text {reference }}(\mathrm{mV})$ & $A_{x}$ & $k_{x}\left(\mathrm{mV}^{-1}\right)$ & $V_{x}^{*}(\mathrm{mV})$ \\
\hline$m_{\infty}$ & -20 & 1.61 & 0.0985 & -23.7 \\
$h_{\infty}$ & -80 & 1.03 & -0.165 & -59.2 \\
$n_{\infty}$ & -20 & 1.55 & 0.216 & -24.3
\end{tabular}



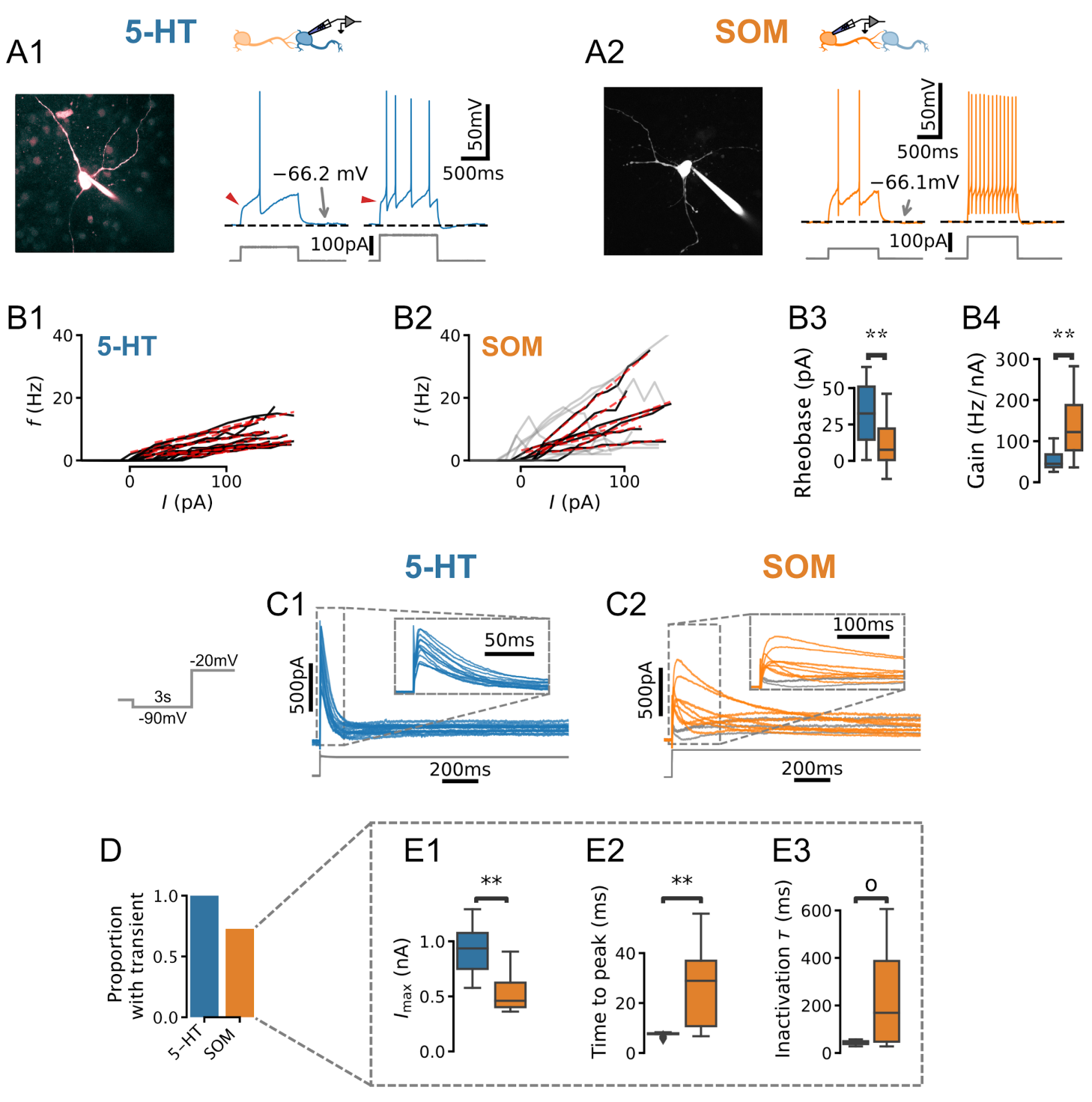

Figure 1 Physiology of DRN neurons. (A, B) Morphology, current steps (A), and spike frequency vs. input ( $f / I$ ) curves $(B)$ of genetically-identified DRN neurons. Non-monotonically-increasing $f / I$ curves (gray) and linear fits to monotonically-increasing curves (red dashed) for $N=17$ 5-HT (B1) and $N=14$ SOM (B2) neurons. (B3) Rheobase of $33.8 \pm 21.0$ pA in 5-HT neurons vs. $11.3 \pm 16.0$ pA in SOM neurons. (B4) Gain of $52.2 \pm 22.2 \mathrm{~Hz} / \mathrm{nA}$ in 5 -HT neurons vs. $87.2 \pm 33.0 \mathrm{~Hz} / \mathrm{nA}$ in $\mathrm{N}=7$ SOM neurons with monotonically-increasing $f / I$ curves. (C) Leak-subtracted whole-cell currents evoked by a depolarizing step. Each trace is one cell; $N=13$ 5-HT and $N=11$ SOM cells. Traces without a transient outward current are shown in gray. (D) Proportion of neurons with a transient outward current by cell type. (E) Quantification of transient outward currents in each cell type. $N=3$ SOM cells without a transient outward current were excluded from analysis, leaving $N=135$-HT and $N=8$ SOM neurons. Annotations reflect Mann-Whitney U-tests. Non-parametric Brown-Forsythe equality of variance tests indicated significantly more variable time to peak $(p=1.11 e-4 ; E 2)$ and inactivation time constant ( $p=1.97 e-4 ; E 3$ ) in SOM cells. 


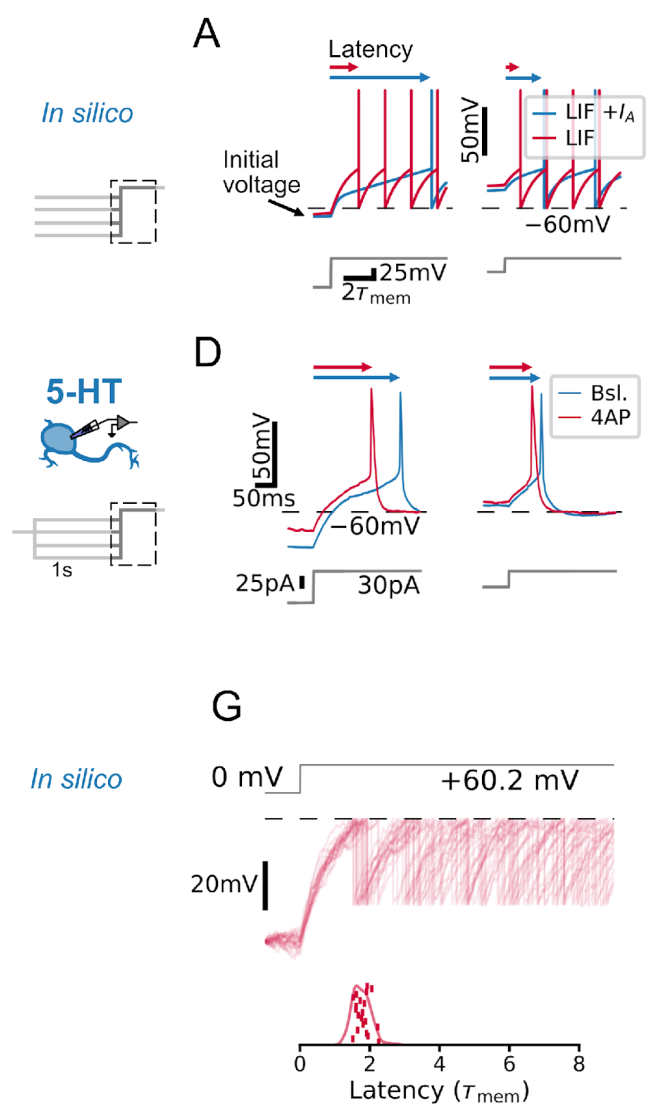

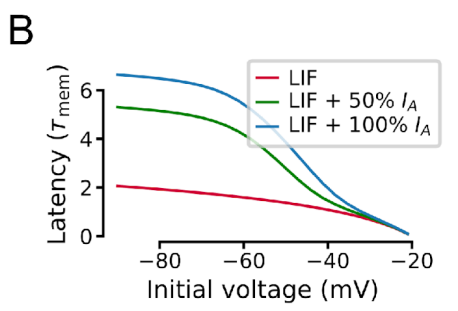

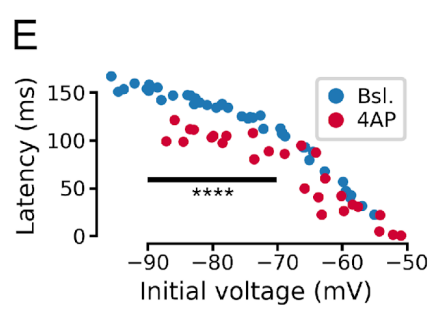

$\mathrm{H}$

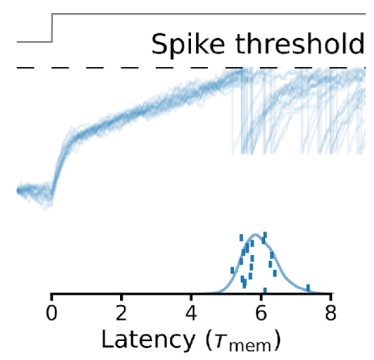

$\mathrm{F}$

C
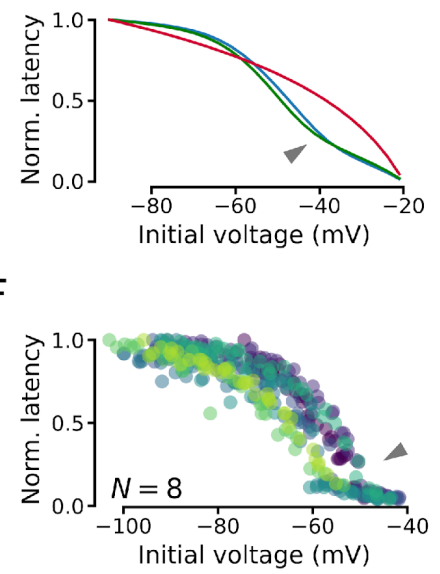

I

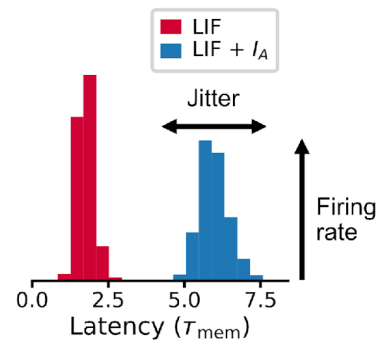

Figure $2 I_{A}$ qualitatively alters the relationship between initial voltage and spike timing. (A-C) A toy model neuron with $I_{A}$ predicts a non-linear effect of voltage history on spike timing in a simple experiment. ( $D-F)$ Experiments in 5-HT neurons fulfill predictions of the toy model. (F) Latency curves for $N=8$ 5-HT neurons, normalized to the maximum latency for each cell. Each colour is one cell. $(G-I) I_{A}$ causes an increase in spike latency and jitter in the presence of noise. Models and input are the same as in A-C. Spike latency histograms for populations of 600 toy neurons are shown in I. The width of the histogram reflects jitter in the timing of the first spike, while the height of the histogram approximates the peak instantaneous firing rate. Note that as jitter increases, the height of the histogram decreases.

\section{$I_{A}$ regulates initial firing rate via a control of spike time jitter}

To develop an intuition for how $I_{A}$ impacts the firing patterns of 5-HT populations, we first created a toy model that captured the effect of this conductance on single cell voltage dynamics (see Methods). In keeping with previous studies, $I_{A}$ introduced a kink in the subthreshold voltage leading up to spike threshold (Getting et al. 1983; Segal et al. 1985; McCormick et al. 1991; Fig. 2A) and increased the latency to the first spike evoked by a square step stimulus, particularly when starting from a hyperpolarized voltage at which $I_{A}$ is free from inactivation (Fig. 2B, C). The relationship between initial-voltage and latency predicted by our toy model was experimentally recapitulated in whole-cell recordings from identified 5-HT neurons (Fig. 2D-F). In particular, the onset of spiking was delayed by hyperpolarization (Fig. 2D), and the magnitude of this effect was significantly reduced by 
the partial pharmacological block of $I_{A}$ with 4-AP $(p=3.2 \mathrm{e}-6$ for initial voltages between -90 $\mathrm{mV}$ and $-70 \mathrm{mV}$; Fig. 2E). We further observed an inflection point predicted by the model in the normalized initial-voltage/latency relationship (Fig. 2F, compare with model prediction in Fig. 2C). In summary, our toy model captured the expected effects of $I_{A}$ in single 5-HT cells.

Next, we used our experimentally-validated toy model to understand how $I_{A}$ impacts the spiking responses of whole neuronal populations. To do this, we simulated the effect of a shared step input to a population of 600 toy neurons each receiving independent background noise (corresponding to naturalistic fluctuations in synaptic inputs). Whereas subthreshold fluctuations yielded time-locked spikes without $I_{A}$ (Fig. $2 \mathrm{G}$ ), they induced spiking with larger jitter across the simulated population when $I_{A}$ was present (Fig. $2 \mathrm{H}$ ). This desynchronizing effect of $I_{A}$ also decreased the peak population rate at the time corresponding to the mean latency (Fig. 2I) since the peak rate corresponds to the coincidence rate from an ensemble of cells with similar properties. Taken together, results from these toy-models revealed a role of $I_{A}$ in regulating the degree of synchronization of a population following sudden inputs, suggesting that $I_{A}$ may regulate the gain of the DRN network to time-varying inputs. This intuition gleaned from this toy model is examined in more detail with optimized GIF models (see below).

\section{Extensions to GIF models are required to capture the excitability of DRN neurons}

We next sought to develop a model able to capture the essential biophysical features of DRN neurons and accurately predict their responses to naturalistic inputs. GIF models offer a flexible modelling framework well-suited to this purpose because they can be trained to accurately reproduce the firing patterns of individual neurons using less than five minutes of electrophysiological data per neuron (Gerstner et al. 2014, Teeter et al. 2018, Paninski et al. 2005, Mensi et al. 2012, Pozzorini et al. 2015). In this framework, individual neurons are described in terms of three core components: 1 ) a passive membrane filter, $\kappa$, which transforms input currents into a subthreshold membrane potential; 2) a stochastic spiking process, which transforms the subthreshold membrane potential into action potentials; and 3) two adaptation mechanisms, namely a spike-triggered current mediating the commonly observed AHP, $\eta$, and change in firing threshold, $\gamma$ (Fig. 3A1, see Methods). These components are described by parameters the values of which are inferred from the electrophysiological data using a combination of least-squares multi-linear regression and gradient ascent of a likelihood function. The flexibility and data-efficiency of this framework lends itself well to capturing the functional properties of single neurons and, by extension, heterogeneous neural populations.

Our results outlined in Figure 2 show that $I_{A}$ regulates spike timing in 5-HT neurons because of its nonlinear subthreshold effects. Foreseeing that the presence of this prominent current may limit the accuracy of canonical GIF models-which are not designed to capture nonlinear subthreshold effects-we first augmented the canonical GIF model (aGIF; Fig. 3A2) with a simplified Hodgkin-Huxley-type model of the subthreshold voltage-dependent currents we recorded in 5-HT neurons (see Methods). As a point of comparison for the aGIF model, we considered the previously-described sodium channel-inactivation GIF model (iGIF; Fig. 3A3), which extends the GIF model of Mensi et al. 
(2012) by adding a non-linear coupling term between the subthreshold voltage and spike threshold (Mensi et al., 2016; see Methods). Although this GIF model extension was initially conceived specifically to capture the influence of subthreshold sodium channel inactivation on firing threshold (hence its name), it has the capacity to account for a wide range of other subthreshold biophysical mechanisms which regulate spiking, notably including, but not limited to, $I_{A}$. Comparing the performance of the more parsimonious aGIF model to that of the iGIF model enabled us to assess whether accounting for additional mechanisms that regulate spiking beyond $I_{A}$ might further improve our DRN neuron models.

To establish comparative GIF model benchmarks across cell types, we carried out whole-cell electrophysiological recordings not only from DRN 5-HT and SOM cells, but also from canonical deep-layer pyramidal neurons of the medial prefrontal cortex (mPFC). For each recording, we applied two distinct instantiations of noisy in vivo-like inputs (see Supplemental Methods, Fig. 3S1), one of which was used to determine the model parameters while the other was reserved for post hoc evaluation of the models' accuracy (i.e., "training" data and "validation" data, respectively; see Fig. 3C). Accuracy was assessed by comparing models with recorded data across cell types in terms of (Fig. 3D): 1) subthreshold voltage changes on training data, $\mathrm{R}^{2}$, and; 2) spike timing on validation data, $M_{d}{ }^{*}$ (where $M_{d}{ }^{*}=1$ is the best possible performance and $M_{d}{ }^{*}=0$ is chance-level; see Methods).

The canonical GIF model predicted both the subthreshold dynamics and spike timing of mPFC pyramidal neurons with high accuracy $\left(\mathrm{R}^{2}=0.431 \pm 0.249 ; M_{d}{ }^{*}=0.783 \pm 0.134\right.$; Fig. 3E), consistent with previous reports on cortical pyramidal neurons (Mensi et al. 2012; Pozzorini et al. 2015; Mensi et al. 2016; Teeter et al. 2018). While our aGIF model slightly better predicted the voltage of $\mathrm{mPFC}$ neurons $\left(\mathrm{R}^{2}=0.544 \pm 0.280, p=0.028\right.$, Fig. 3E1), this did not translate into more accurate spike predictions $\left(M_{d}{ }^{*}=0.743 \pm 0.180, p=0.710\right.$, Fig. 3E2), consistent with the observation that $I_{A}$ is not a significant conductance recorded from the cell body of mPFC pyramidal neurons (Fig. 3S2 and see Dong et al. 2003; 2005). On the basis of spike timing prediction, the canonical GIF model thus offered the most parsimonious account of the behaviour of mPFC neurons.

With this point of comparison established, we next quantified the performance of each of our candidate GIF models (GIF, aGIF and iGIF) in 5-HT neurons. As previously intuited, the canonical GIF model performed rather poorly in 5-HT neurons (Fig. 3F), predicting < $15 \%$ of the variance of the subthreshold voltage $\left(\mathrm{R}^{2}=0.128 \pm 0.135\right)$ and achieving $M_{d}{ }^{*}$ scores less than half of those observed in $\mathrm{MPFC}$ neurons $\left(M_{d}{ }^{*}=0.352 \pm 0.118\right)$. This indicates that the passive membrane filter and adaptation mechanisms included in the canonical GIF model were insufficient to capture the behaviour of 5-HT neurons. By augmenting the GIF model with our experimentally-constrained model of $I_{A}$, the aGIF model not only better predicted the voltage $\left(\mathrm{R}^{2}=0.301 \pm 0.200, p=1.96 \mathrm{e}-4\right.$; Fig. 3F1) but also the spike timing ( $M_{d}^{*}=0.481 \pm 0.148, p=0.001$; Fig. 3F2) of 5-HT neurons. While the iGIF model exhibited 
a similar improvement in spike timing predictions over the GIF model $\left(M_{d}^{*}=0.536 \pm 0.154\right.$, $p=5.89 \mathrm{e}-4)$, it did not significantly outperform the aGIF model ( $p=0.644$; Fig. 3F2). Thus, by adding $I_{A}$ to the subthreshold and spiking mechanisms of the GIF model, the aGIF model best accounts for the biophysical mechanisms responsible for shaping the responses of 5-HT neurons to in vivo-like inputs. Turning to the other main cell type of the DRN, we next analyzed the performance of each model in SOM cells (Fig. 3G). In these cells, the canonical GIF model produced highly accurate predictions $\left(\mathrm{R}^{2}=0.600 \pm 0.238\right.$ and $M_{d}{ }^{*}=0.818 \pm$ 0.149 ), consistent with its high performance previously reported for cortical GABAergic neurons (Mensi et al. 2012; Teeter et al. 2018). Nonetheless, the iGIF achieved small but significant performance gains $\left(M_{d}{ }^{*}=0.892 \pm 0.094, p=0.004 \mathrm{vs}\right.$. aGIF and $p=0.003$ vs. GIF; Fig. 3G2), leading us to select it as our model of SOM neurons. 
bioRxiv preprint doi: https://doi.org/10.1101/2021.06.25.449907; this version posted June 27, 2021. The copyright holder for this preprint (which was not certified by peer review) is the author/funder, who has granted bioRxiv a license to display the preprint in perpetuity. It is made available under aCC-BY-NC-ND 4.0 International license.

A1 Model 1: GIF

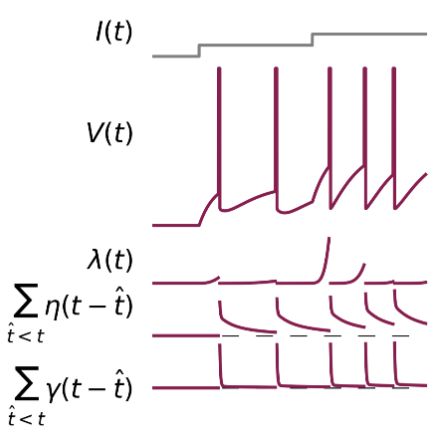

A2 Model 2: aGIF

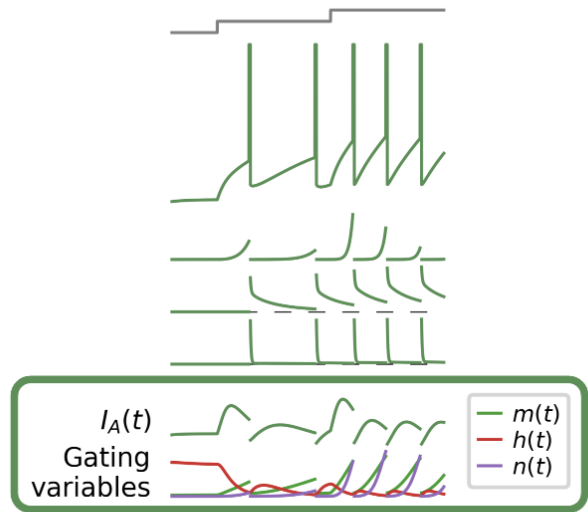

A3 Model 3: iGIF

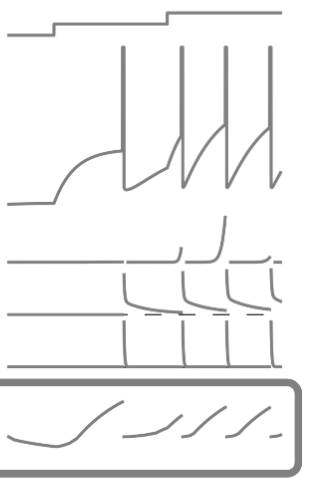

B

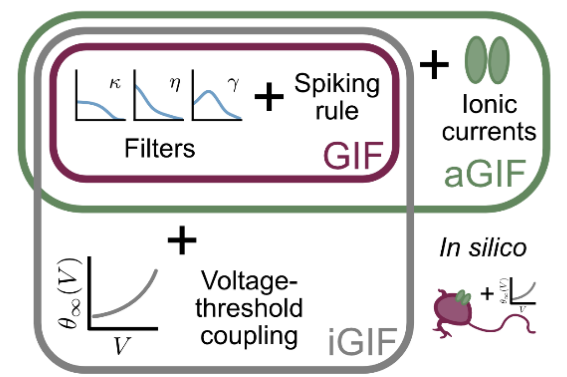

C

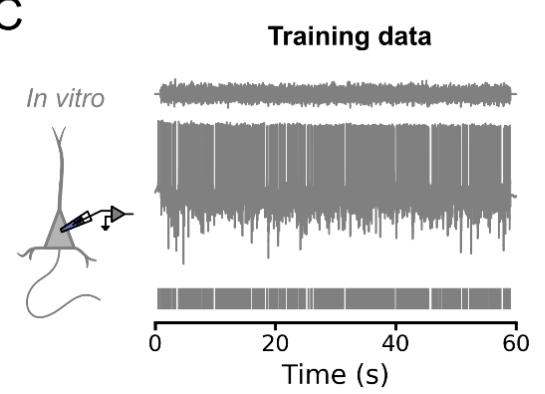

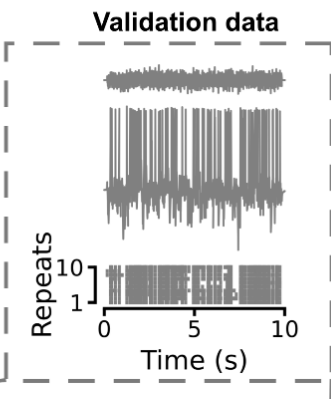

D3 SOM

\section{D1 mPFC L5}

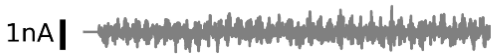

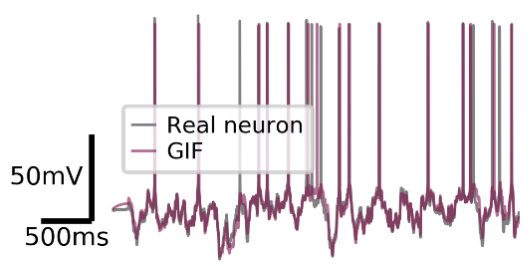

D2 $5-\mathrm{HT}$
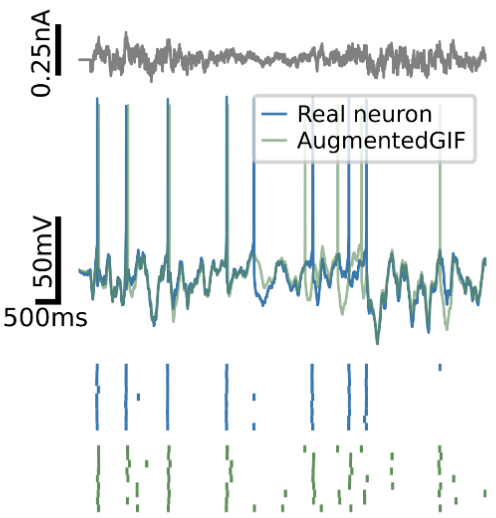

F1

E1

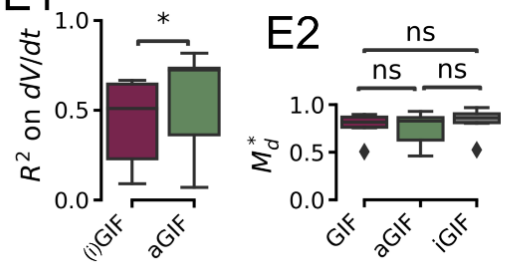

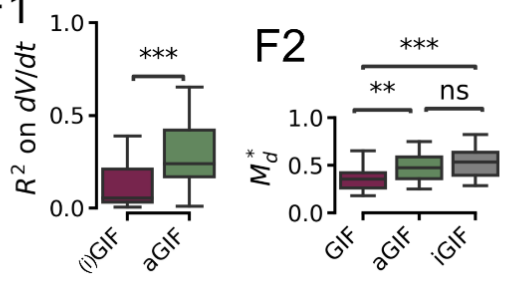

$0.5 \mathrm{nA}$

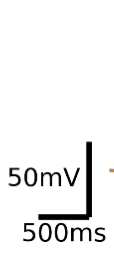

G1
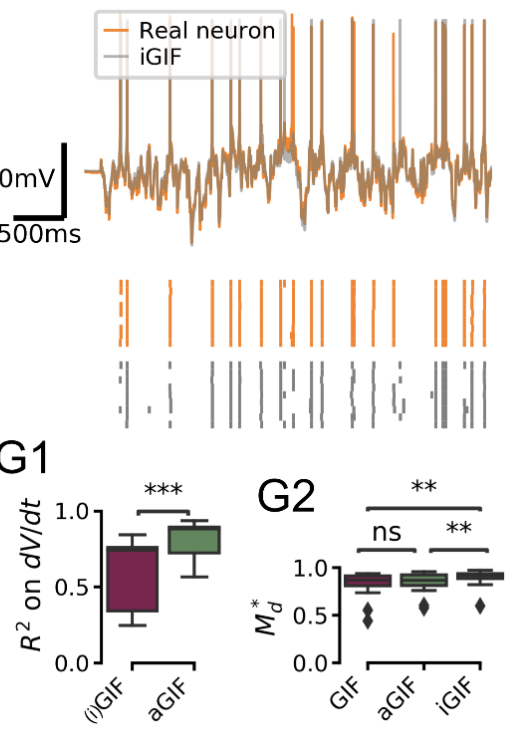
Figure 3 Single neuron models accurately predict the subthreshold voltage and spike times of DRN neurons. (A) Components of candidate single neuron models: $\lambda$ intensity of stochastic spike-generating process; $\eta$ spike-triggered current (positive values indicate a hyperpolarizing current); $\gamma$ spike-triggered threshold movement. (B) GIF model extensions. (C) Representative experiment used to train and validate neuron models. Training set consists of repetitions of $60 \mathrm{~s}$ of frozen Ornstein-Uhlenbeck (OU) noise and the validation set consists of repetitions of a different frozen OU noise stimulus lasting $10 \mathrm{~s}$ (only one repetition is shown). (D) Representative validation data and model predictions for each cell type. OU noise input current (top), recorded and predicted voltage traces (middle), and recorded and predicted spike times across all repetitions of the validation stimulus. Stimulus parameters were adjusted for each cell type, see Fig. 3S1. (E-G) Quantification of model performance in terms of $R^{2}$ on the training subthreshold voltage derivative and on the validation

spike-train similarity metric $M_{d}{ }^{*}$. GIF and iGIF models have the same subthreshold performance because the subthreshold components of these models are identical (see Methods). Benchmarks are for models fitted to $N=185$-HT, $N=14$ SOM, and $N=7$ mPFC neurons.

\section{Prominent moving threshold in 5-HT neurons}

Our model selection approach identified the most salient components required to capture the input-output functions of individual neurons and allowed us to identify functional differences across cell types. 5-HT neurons were distinguishable from SOM and MPFC cells by their long membrane time constants (Fig. 4A and 4S1A) and by the presence of conspicuously potent and protracted adaptation mechanisms (Fig. 4B-D). Indeed, in addition to evoking a characteristically large and prolonged adaptation current (Fig. 4C), action potential firing in 5-HT neurons produced a substantial and long-lasting increase in firing threshold (Fig. 4D). In contrast, SOM neurons most often displayed either negligible or even depolarizing spike-triggered currents (Fig. 4B,C) that may underlie the burst firing patterns often observed in this cell type (Fig. 1S2). These observations derived from the parameters of GIF models are not only consistent with our experimental characterization (Figs 1-3), but significantly expand it. Thus, 5-HT neurons are characterized by slow membrane dynamics, $I_{A}$, and particularly prominent adaptation mechanisms. 
A

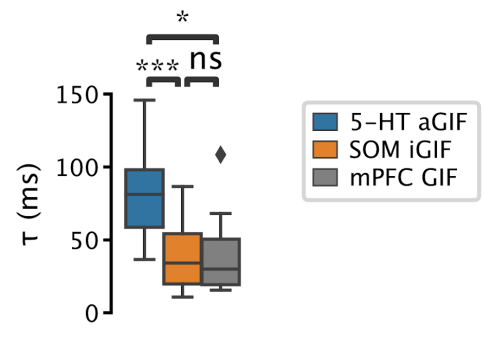

C

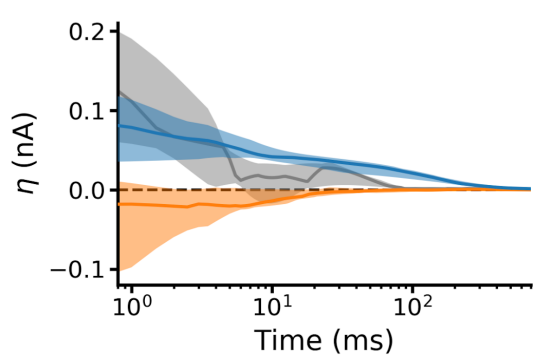

B

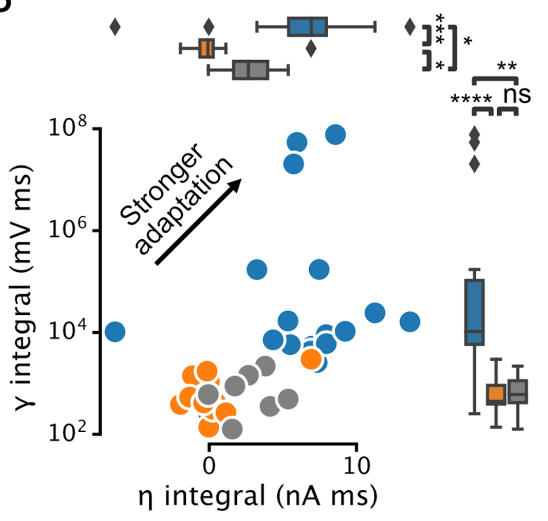

D Threshold movement

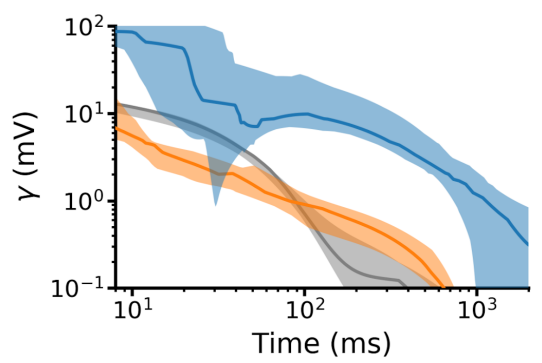

Figure 4 5-HT neurons are distinguished by slow membrane time constants and potent adaptation. (A) Using the features from the best-performing GIF model variant for each cell type (legend), passive membrane time constant. (B) Spike adaptation features: potency of AHP-mediated (spike-triggered current $\eta$ integral) and AHP-independent (spike-triggered threshold movement $\gamma$ integral) adaptation. $(C, D)$ Comparison of model filters. Presented as median (lines) and interquartile range (bands). Note the long-lasting adaptation currents ( $C$; positive values indicate hyperpolarizing current) and threshold movements (D) of 5-HT neurons.

\section{Preferential sensitivity of 5-HT neuron population to the onset of sudden inputs}

The development and validation of accurate single-cell models allowed us to identify the population-level computations operating in the DRN. We took advantage of the one-to-one correspondence between our GIF models and real neurons to construct synthetic populations with realistic neuron-to-neuron heterogeneity by sampling from banks of single-cell models (Fig. 5A). In response to step increases of synaptic-like inputs delivered to the entire population (Fig. 5B left), the population firing rates (in Hz/neuron; Fig. 5B right) of 5-HT, SOM, and mPFC neurons (Fig. 5C) transiently increased before relaxing to a significantly lower stationary level. Strong inputs did not produce oscillations in the population firing rates, likely because of population heterogeneity (Fig. 5S3; Naud and Gerstner 2012; Mejias et al. 2012; 2014; Tripathy et al. 2013). The transient and stationary 
parts of the population input-output functions were approximately rectified linear functions (Fig. 5S3) which we summarized and plotted as the time-varying slope (i.e., gain; Fig. 5D). While the gain of the transient response was greater than that of the stationary response in all three cell types, the ratio of transient to stationary gain was substantially

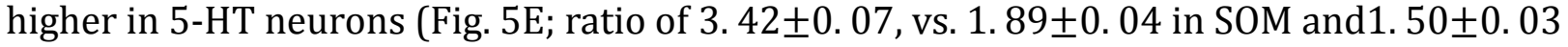
in MPFC; $p<0.001$ in each case). This marked response of 5-HT cells occurred quickly, in the first $100 \mathrm{~ms}$ after the onset of the step. Thus, despite 5-HT neurons being characterized by slow membrane time constants, their population activity provided a remarkably strong encoding of the onset of step synaptic inputs.

We next considered the underlying mechanisms giving rise to the distinctive time-dependent gain of 5-HT neurons. We found that the characteristically strong spike-triggered adaptation of 5-HT neurons (spike triggered hyperpolarizing adaptation current and threshold movement shown in Fig. 4) contributed to the observed relaxation of the population response to a lower stationary level: grafting the weak adaptation from SOM neuron models onto 5-HT models dramatically reduced the ratio of transient to stationary gain, and vice-versa (Fig. 5F). These findings are consistent with previous models in other cell types showing that spike-triggered adaptation reduces the sensitivity of neural populations to input changes over long timescales (Ermentrout 1998; Benda and Herz 2003; Naud and Gerstner 2012). Therefore the preferential sensitivity of 5-HT neuron populations to sudden changes in synaptic inputs is a natural consequence of strong adaptation at the single neuron level. 
A

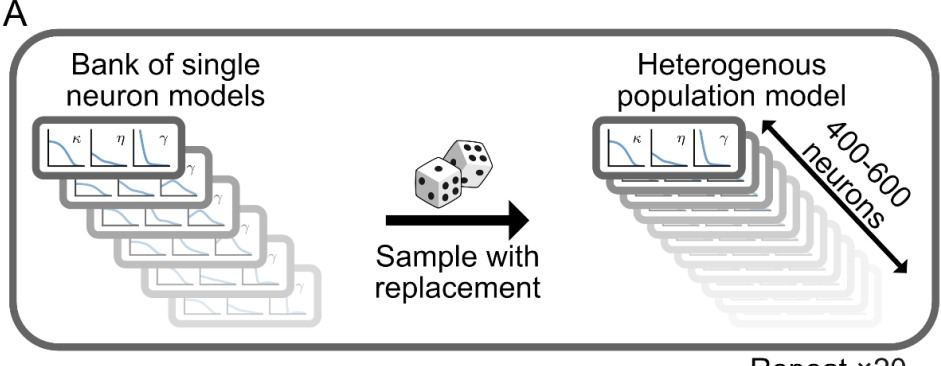

Repeat $\times 20$
B $\quad \begin{aligned} & \text { Mean } \\ & \text { input }\end{aligned}$

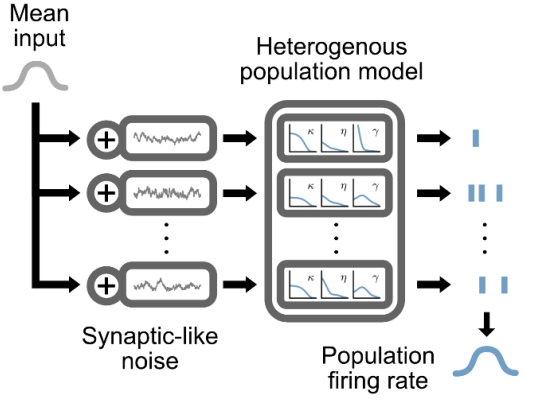

C1

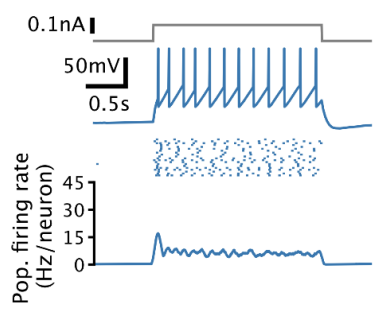

C2 SOM in sillico

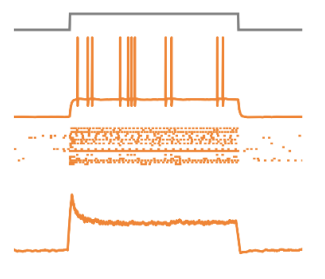

C3 mPFC in sillico
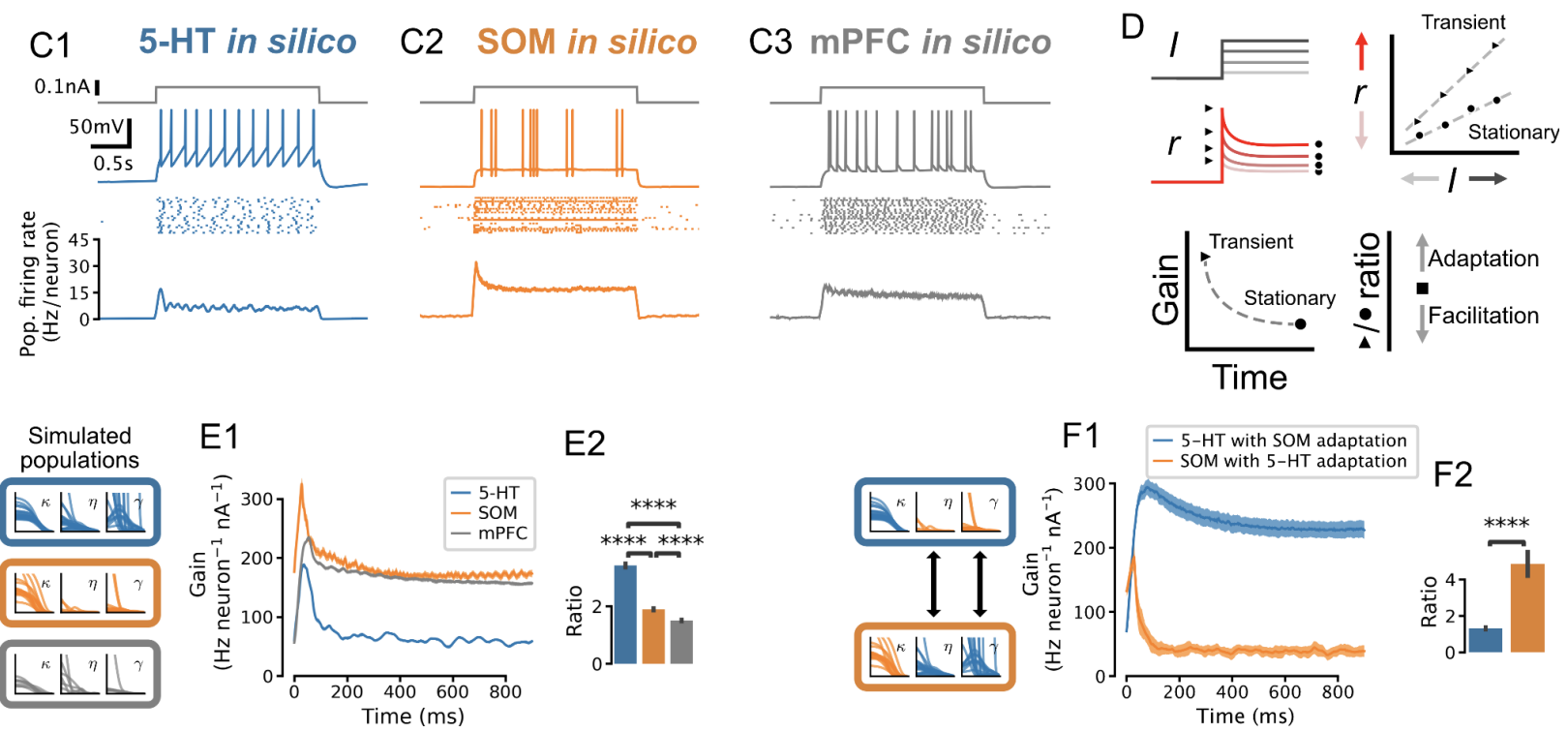

E2

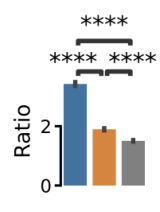

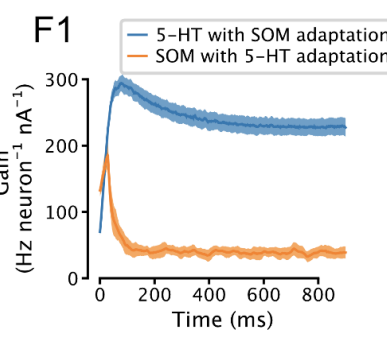

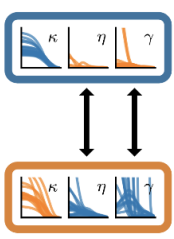

F2

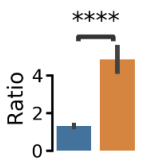

Figure 5 Adaptation mechanisms cause a higher gain of the transient vs stationary population response. (A) Generation of heterogeneous population models from experimentally-constrained single neuron models. (B) Schematic of population simulations. Spikes from individual neuron models in the simulated population are added together to produce a population firing rate. (C) Population responses to input step. From top in each column: stimulus (gray); sample voltage trace; spike raster of first 20 neurons; mean population firing rate across 20 independent simulations. (D) Schematic for quantifying the time-varying population input-output function for both the transient and the stationary components of the response. An input-output function is calculated for the population response at each time point after the input step. The slope of each input-output function (gain) is then plotted as a function of time since the step onset. The ratio of the maximum gain to the minimum gain is a measure of the relative amount of population adaptation. (E) Time-resolved gain of step input responses across cell types following the approach shown in D. (F) Time-resolved gain of 5-HT populations with the adaptation parameters of SOM neurons (blue) and of SOM populations with adaptation parameters of 5-HT neurons (orange).

\section{Feedforward inhibition and $I_{A}$ control 5 -HT output gain of the DRN}

Apart from the strong adaptation mechanisms of 5-HT neurons, two other mechanisms have the potential to dynamically modulate the 5 -HT output from the DRN: $I_{A}$ in 5 -HT 
neurons and the feed-forward inhibition (FFI) enacted by local DRN interneurons (Zhou et al. 2017; Geddes et al., 2016). To examine the contributions of these two mechanisms, we first connected our existing SOM population models to 5-HT population models using experimentally-constrained $\mathrm{GABA}_{\mathrm{A}}$ receptor-mediated synaptic conductances (see Methods and Fig. 5S4).

To dissect the contribution of $I_{A}$ in shaping population responses in this connected DRN network, we applied the same inputs to both 5-HT and SOM neuron populations and examined 5-HT neuron population dynamics (as in Fig. 5) while varying the maximal conductance of $I_{A}$ (in 5-HT neurons). The gain of the transient component of the 5-HT response increased markedly when the conductance of $I_{A}$ was set to zero (Fig. 6A), while increasing the potency of $I_{A}$ substantially dampened and broadened the population response to fast inputs, reminiscent of $I_{A}$ 's modulation of spike timing jitter observed in our toy model (Fig. 2I-K). These simulations thus show that $I_{A}$ substantially regulates the gain of the transient component of DRN 5-HT output evoked by sustained inputs, with negligible effects on the gain of the slower stationary component.

Previous work has shown that glutamatergic excitatory inputs from the PFC make strong mono-synaptic contacts onto both DRN 5-HT and GABAergic neurons, triggering a classic FFI. Intriguingly, the PFC axonal inputs onto these two cellular elements of the DRN are functionally distinct inasmuch as the PFC synapses onto GABAergic neurons are far more sensitive to endocannabinoid neuromodulation than those onto 5-HT neurons (Geddes et al., 2016). The computational role of this differential sensitivity to neuromodulation is currently unknown. We began by determining the role of the DRN FFI per se by comparing the responses of 5-HT neuron population dynamics with or without SOM cells (Fig. 6B). Including FFI onto 5-HT neurons substantially dampened the overall response of the 5-HT population to synaptic inputs, while still sustaining the preferential encoding of the early phase of sudden inputs (Fig. 6B2). While introducing FFI did decrease the gain ratio, this decrease was quantitatively smaller than the differences between 5-HT neurons and other cell types shown in Fig. 5E and the effect of changing $I_{A}$ shown in Fig. 6A, Fig. 6B2. We next directly simulated the effects of endocannabinoid modulation of excitatory input to the DRN observed experimentally (Geddes et al., 2016) by weakening the strength of the input to SOM neuron populations by $30 \%$ while leaving that to 5 -HT neurons intact. By favoring the direct monosynaptic excitation of 5-HT neurons by preferentially diminishing the glutamatergic drive of SOM neurons, this neuromodulation led to an increase in the overall gain of the DRN that was unexpectedly apparent across the entire duration of the response to step inputs (i.e., no change in the gain ratio, Fig. 6B2). Thus, the target-specific endocannabinoid-mediated modulation of PFC excitatory drive in DRN exerts a normalizing role by increasing the overall gain of 5-HT output evoked by synaptic inputs without altering its preferential encoding of changes in input, which is emerging as a cardinal feature of DRN network dynamics.

Our electrophysiological recordings showed that excitability heterogeneity is a salient feature of the SOM DRN neuron population. Our modeling approach allows us to specifically examine the role of this cellular heterogeneity in shaping the output of the DRN by 
comparing our DRN model (Fig. 6C) to an alternative homogenized version in which the parameters of SOM neurons were set to fixed values (Fig. 6D). Thus, while FFI with an experimentally-determined degree of heterogeneity mainly imposed a reduction of the slope of the input-output function (i.e., divisive inhibition), homogeneous FFI mainly shifted the input-output function of the transient component of the population response to the right (i.e., subtractive inhibition; Fig. 6E). This subtractive feature can be traced back to a strong non-linearity in the input-output functions of homogenized SOM neuron populations (compare Fig. 6E1 and Fig. 6F1). In the case of the stationary component, both heterogeneous and homogenized DRN models implemented divisive inhibition (Fig. 6F). Therefore, we conclude that heterogeneity among GABAergic neurons implements divisive inhibition. 
bioRxiv preprint doi: https://doi.org/10.1101/2021.06.25.449907; this version posted June 27, 2021. The copyright holder for this preprint (which was not certified by peer review) is the author/funder, who has granted bioRxiv a license to display the preprint in perpetuity. It is made available under aCC-BY-NC-ND 4.0 International license.

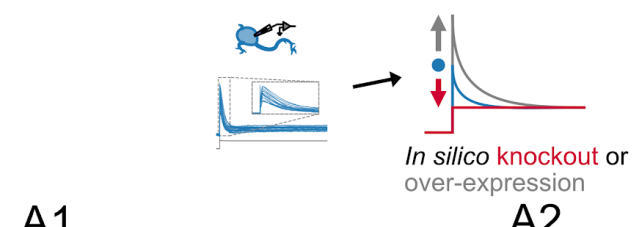

A1
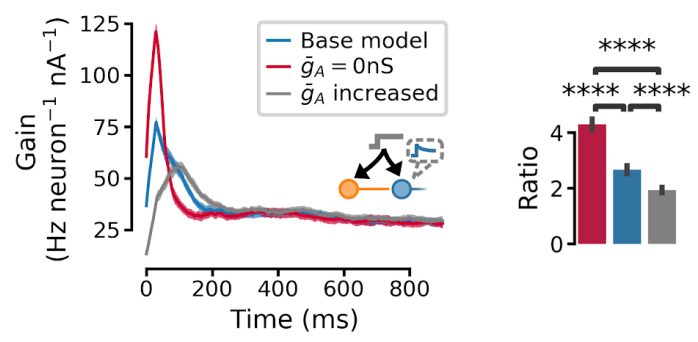

Heterogenous SOM population
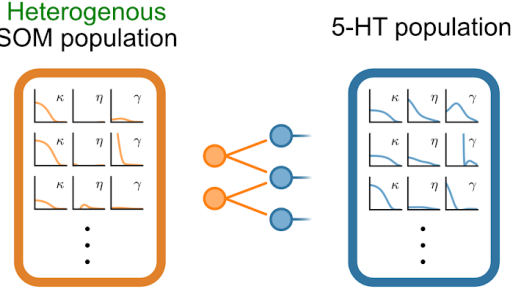

C1

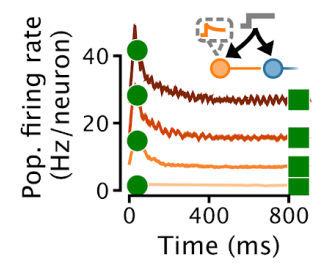

$\mathrm{C} 2$

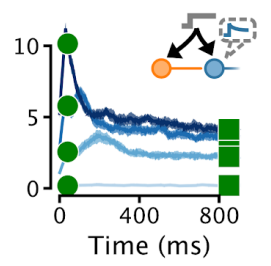

\section{O Transient}

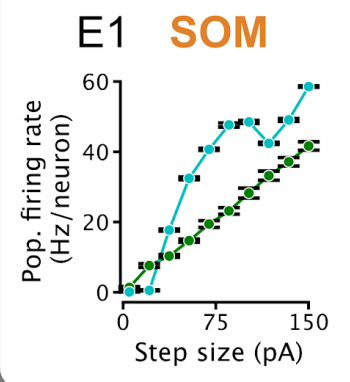

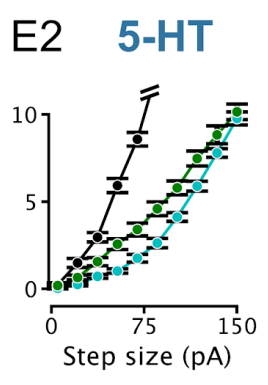
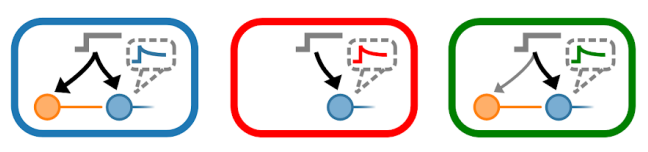

B1

B2
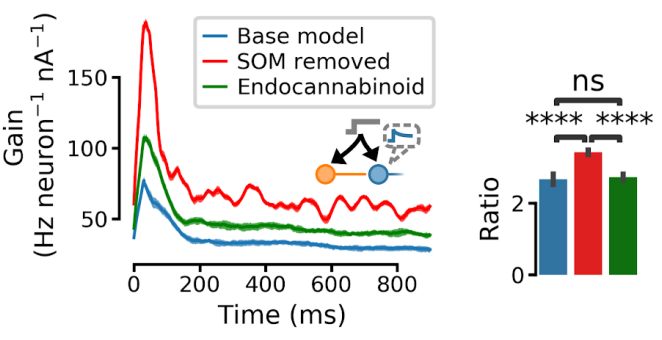

Homogenous

SOM population
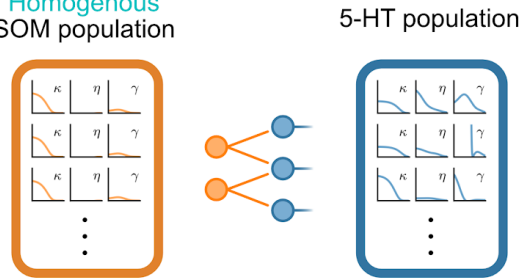

D1

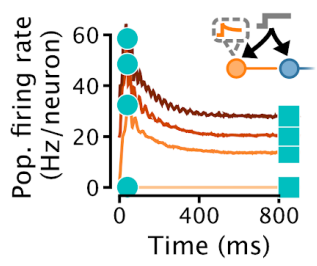

D2

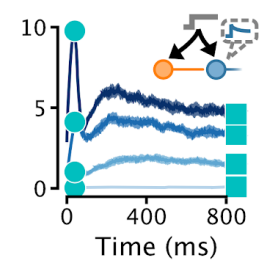

\section{Stationary}

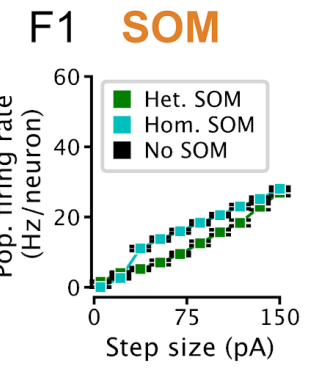

F2 $5-\mathrm{HT}$

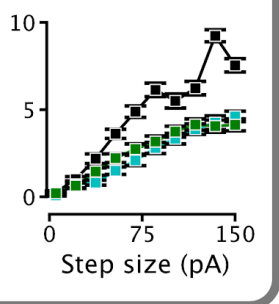

Figure 6 Effect of $I_{A}$ density, feedforward inhibition, and heterogeneity of SOM neurons on the 5-HT neuron population response. Network input is the same set of step stimuli as in Fig. 5D-F. (A) Increasing $I_{A}$ reduces adaptation by selectively suppressing the early part of the response to sudden inputs, and vice-versa. (B) Gain curves with normal feedforward inhibition (blue), with reduced input strength onto the inhibitory population (green), or without inhibition (red). Reduced input strength onto the inhibitory population (green) simulates the effect of endocannabinoid input (Geddes et al., 2016). (A2,B2) Ratio of peak to steady-state gain. (C) Population firing rates of SOM and 5-HT neurons in a network in which both populations are heterogenous. (D) Population firing rates of SOM and 5-HT 
neurons in a network in which all SOM neurons are identical. Effects of homogeneous (cyan) or heterogeneous (green) SOM populations on the population input-output functions for the transient (E) and stationary $(F)$ components of the response (see square and circle markers in $C$ and $D$ ). Note that the input-output function of the heterogenous SOM population is approximately linear, whereas that of the homogenous population is not (E1). Relative to the input-output functions of a 5-HT population receiving no feed-forward inhibition, the effect of the heterogenous SOM population is divisive, but the effect of the homogenous SOM population includes a strong subtractive component (F2).

\section{5-HT neurons linearly encode the temporal derivative of inputs to the DRN}

Adaptation plays a critical role in implementing temporal derivative encoding in sensory systems (Lundstrom et al. 2008; Pozzorini et al. 2013), but has not been ascribed a similar role in neuromodulatory systems such as the DRN. To determine whether the DRN also supports this computation, we parameterized the rate of change of DRN inputs by applying ramp stimuli with variable slopes (i.e., derivatives; Fig. 7A and B). Remarkably, the peak 5-HT neuron population firing rate linearly reported the slope of the ramps, while SOM neuron populations were essentially slope-insensitive (Fig. 7C and D). We further found that this linearity was conditional on the presence of slightly depolarizing background input ( $\geq 20$ pA, Fig. 7E). The potent adaptation mechanisms of 5-HT neurons play a key role in mediating this linear encoding of input derivative, since reducing the strength of adaptation reduces linearity across a wide range of input baselines (Fig. 7F-G). Removing $I_{A}$ extended the range of background input where linearity is observed (Fig. $7 \mathrm{H}$ ). In summary, we found that the presence of strong spike-frequency adaptation in 5-HT neurons causes the DRN to signal the rate of change of its input to its brainwide targets, but that this core computation is progressively suppressed when a state of hyperpolarization engages $I_{A}$. 
A
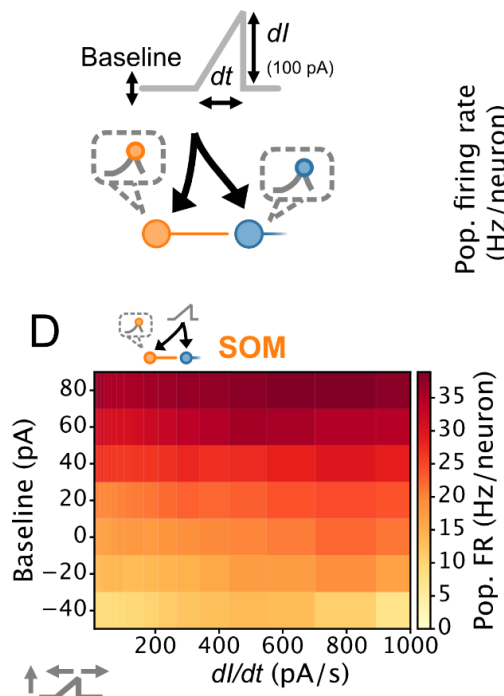
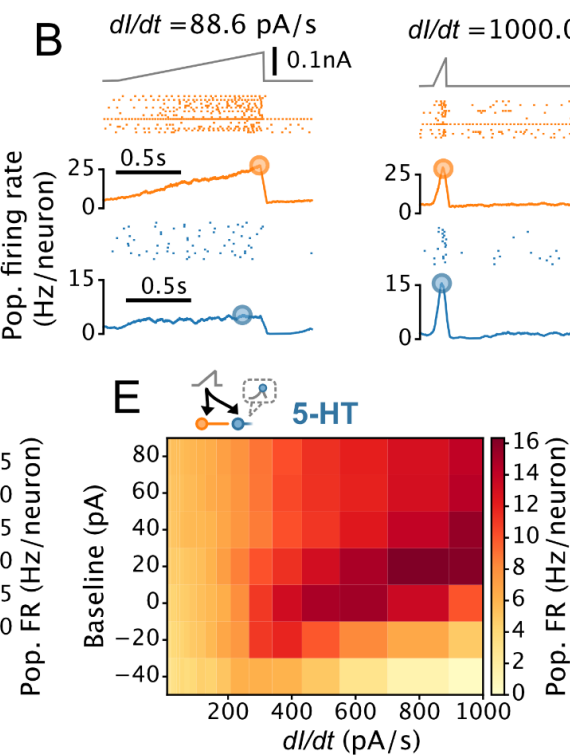
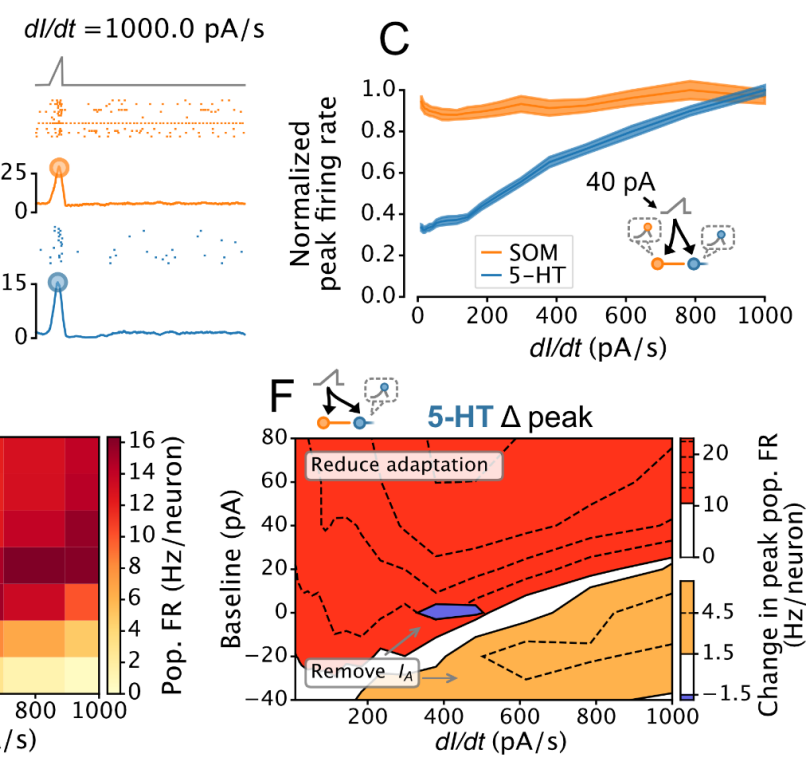

G

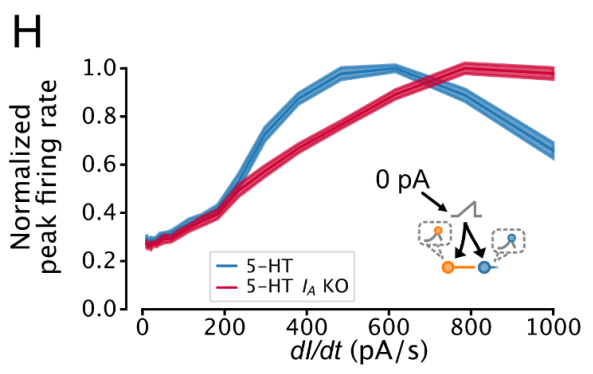

Figure 7 DRN 5-HT neuron population output conditionally encodes the temporal derivative of its input. (A) Design of simulations. A ramp stimulus with an adjustable baseline and slope (derivative) is applied to the same network models as in Figs. 5 and 6 and the peak firing rate of each population is extracted. (B) Representative simulated input (top), SOM neuron population activity (middle), and 5-HT neuron population output (bottom). (C) With a baseline input of $40 \mathrm{pA}$, 5-HT neuron population output is approximately linearly related to the derivative of the ramp input, but that of the SOM neuron population is not. (D,E) Peak firing rates (FRs) of SOM neuron populations depend mainly on input baseline, while those of 5-HT populations depend on interacting effects of input baseline and slope. Panel $C$ shows normalized data from the $40 \mathrm{pA}$ rows in D (orange) and $E$ (blue). (F) Effect of reducing 5-HT neuron adaptation (following the approach from Fig. $5 F$ ) or removing $I_{A}($ following the approach of Fig. 6A) on peak 5-HT neuron population firing rate. (G) Effect of reducing adaptation in 5-HT neuron models visualized at a 40pA baseline. Note that 5-HT output no longer linearly encodes $d I / d t$ when adaptation is reduced. (H) Effect of removing $I_{A}$ from 5-HT neuron models visualized at a $0 \mathrm{pA}$ baseline. Note that 5-HT output approximately linearly encodes $d I / d t$ when $I_{A}$ is removed. 


\section{Discussion}

Here, we sought to characterize the computational properties of the DRN using a bottom-up approach grounded in experimentally-constrained models of the two most abundant cell types in this region: 5-HT and SOM GABA neurons. Consistent with, and extending, previous work, we found that 5-HT neurons were relatively homogeneous and characterized by potent spike-frequency adaptation (Fig. 1) and by the presence of a strong A-type potassium current (Fig. 2), while SOM neurons displayed a considerably more heterogeneous excitability profile (Fig. 1 and Fig. 1S2). Extensions to classical generalized integrate-and-fire (GIF) models (Mensi et al., 2012, Pozzorini et al., 2015) to capture the non-linear subthreshold effects of $I_{A}$ observed in 5-HT neurons were required to adequately capture the spiking response of 5-HT neurons to naturalistic stimuli (Fig. 3). This work introduces a new approach to capturing such non-linear subthreshold effects in the form of the aGIF model, which augments the GIF model of Mensi et al. (2012) with experimentally-constrained Hodgkin-Huxley style currents, simultaneously improving model performance and interpretability. Inspecting the parameters of the best-performing GIF models revealed that the substantial spike-frequency adaptation observed in 5-HT neurons is not fully explained by their distinctively large AHPs, and is partly mediated by a previously undescribed dynamic spike threshold (Fig. 4). This model-based approach allowed us to probe causal relationships between specific excitability features and population computations. Thus, we found that the prominent adaptation mechanisms in 5-HT neurons regulated DRN population responses to synaptic inputs (Fig. 5), that $I_{A}$ conductance suppressed the response to sudden inputs, and that heterogenous FFI had a divisive rather than subtractive effect on gain (Fig. 6). By further exploring DRN population dynamics, our simulations demonstrated that 5-HT, but not SOM, neurons linearly reported the derivative of their synaptic inputs (Fig. 7). In summary, this work points to a new computational role for the DRN in encoding the derivative of its inputs, and identifies specific cellular and network mechanisms that give rise to this computation and modulate its expression. These results raise important questions about how the selective responses of the DRN to changing synaptic inputs might support its role in guiding animal behavior in response to a changing environment.

\section{Need for a hybrid biophysical-simplified methodology}

The computational and statistical modeling methodology presented here was designed to bridge the gap between specific biophysical mechanisms and network level computation. Closing this gap has also been the target of complex biophysical simulations, motivated by the hope to create tools for testing disease-related treatments and for untangling the computations performed by large neural networks (Markram 2006; Billeh et al. 2020). Preserving the accuracy and identifiability of simpler approaches (Gerstner and Naud 2009; Mensi et al. 2012; Pozzorini et al. 2013; Teeter et al. 2018), the “augmented GIF" model developed here explicitly incorporates the most important biophysical features of 5-HT neurons, allowing us to probe their contributions to network-level computation by altering or removing the corresponding model components during network simulations. While the 
aGIF framework was developed here to capture the effects of inactivating subthreshold potassium currents in 5-HT neurons, it lends itself equally well to capturing the effects of other subthreshold voltage-gated currents. We note that, as in other methods based on linear regression of nonlinear ion channel dynamics (Huys and Paninski 2007), adequate experimental estimates of the kinetic features of the conductance at play must be available to be inserted in the aGIF. Altogether, this expanded modeling framework adds to a toolset of computational approaches for interrogating the role of particular microcircuit motifs (e.g., FFI) or excitability features (e.g., spike-triggered adaptation) in shaping network computations, while lending itself to more elaborate inference methodologies (Gonçalves et al. 2020).

Could the dynamical features identified here have been captured by a simpler modeling framework? Two closely-related approaches that we have not considered here are linear-nonlinear (LNL) and generalized linear models (GLMs), which are trained using only the spike output and external input to each cell and do not consider the subthreshold voltage (Pillow et al. 2006; Pillow et al. 2008). Despite the fact that the GLM approach was not possible here given the very low firing rates of 5-HT neurons and the large number of action potentials required for accurate characterization in the absence of information about the subthreshold voltage, it is worth asking whether GLMs could in principle capture the network-level properties of 5-HT signaling. For instance, the role of spike-triggered adaptation in conveying preferential sensitivity to suddenly changing inputs arises in GLMs (Naud and Gerstner 2012), but the state-dependence of the input derivative sensitivity identified in 5-HT neurons (Fig. 7) could not have been captured by a GLM implementation. In summary, the GIF framework provides a more solid foundation for network modeling than LNL- or GLM-based approaches for cell types with very low firing rates or highly state-dependent output.

\section{Network level role of $I_{A}$ current}

Previous modeling work has implicated $I_{A}$ in controlling the sensitivity of the stationary response to sustained inputs (Connor and Stevens 1971; Connor et al. 1977; Drion et al. 2015). These studies contrast with our findings, which implicate this current in the control of the transient component but show almost no effect on the stationary component of the response. This discrepancy can be explained by noting that the AHPs of 5-HT neurons (and thus of our computational model) do not reach the hyperpolarized potentials required to free $I_{A}$ from inactivation (Fig. 1 and Fig. 1S1), in contrast to the model of Connor et al. (1977). As a result, $I_{A}$ remains mostly inactivated during sustained inputs, and the stationary response is mostly regulated by the interplay between spike-triggered adaptation and the strength of the input. Other factors such as a shift in the activation and/or inactivation curves (e.g., by neuromodulators) are expected to influence how $I_{A}$ controls the transient and stationary components of the response. Finally, it is interesting to note that $I_{A}$ is also highly expressed in the dendrites of cortical neurons, where it may have an analogous function (Hoffman et al. 1997; Harnett et al. 2013; Ujfalussy et al. 2018; 
Payeur et al. 2019). Our results hint at a possible general role of $I_{A}$ in suppressing transient responses to sustained inputs in the midbrain, cortex, and other systems.

\section{5-HT neuron heterogeneity}

5-HT neurons are not all alike in every respect: recent experimental work has uncovered molecular, electrophysiological (Calizo et al., 2011), developmental, and anatomical (Commons et al., 2015, Ren et al., 2018) differences among 5-HT neurons across raphe nuclei and within the DRN (reviewed in Okaty et al., 2019). Most relevant to our work are previously reported quantitative differences in the excitability of serotonin neurons located in the dorsomedial DRN, ventromedial DRN, and median raphe nucleus (Calizo et al., 2011). These observations suggest that the predictions made by our model, which was fitted primarily to serotonin neurons from the ventromedial DRN, may agree qualitatively but not quantitatively with the behaviour of 5-HT neuron ensembles in these areas. While there is not yet any evidence that serotonin neurons in different parts of the serotonin system perform qualitatively different computational operations, this remains an intriguing possibility for future work.

\section{Heterogeneous properties of SOM neurons ensure divisive inhibition}

How the heterogeneity of excitability influences the response properties of neuronal populations depends on a number of factors. Specifically, we and others (Mejias and Longtin 2014) have argued that heterogeneity of feedback inhibition (and of principal cells) implements a divisive effect on the stationary part of the population input-output function. For feedforward inhibition, a divisive effect on the gain of stationary input-output functions is expected in naturalistic conditions (Mejias, Payeur, Selin, Longtin 2014). The findings outlined here further support these theoretical results by showing that the heterogeneous feedforward inhibition remains divisive on the transient part of the response. Divisive inhibition has been proposed to be essential to counteract strong excitation so as to maintain activity within an adequate dynamic range (Chance and Abbott 2000, Ferguson and Cardin, 2020), and it is expected that brain circuits will harness cellular and circuit-level mechanisms to tune their sensitivity to relevant inputs while maintaining overall stability. This point is germane to 5 -HT neurons given their position at the confluence of many excitatory input streams (Weissbourd et al., 2014, Dorocic et al., 2014, Ogawa et al., 2014, Zhou et al., 2017, Ren et al., 2018; Geddes et al., 2016). Thus, while the exact behavioral function of the 5-HT system is still unclear, uncovering important components of its gain control mechanisms might provide useful hints about how it integrates its multifold inputs.

\section{Neuromodulation of neuromodulation}

Neuromodulators can dynamically reconfigure information processing in neural circuits that are otherwise anatomically fixed (Marder 2012, Tsuda et al., 2021). While 5-HT is considered to be a neuromodulator, the DRN network is itself under neuromodulatory influence, both from distal (e.g., locus coeruleus or ventral tegmental area) or local (e.g., endocannabinoids, 5-HT itself) sources (Baraban and Aghajanian 1981; Aman et al. 2007; 
Weissbourd et al., 2014; Geddes et al., 2016). Whereas previous work has outlined defined cellular metrics (e.g., changes in release probability or direct membrane depolarization/hyperpolarization that are modulated by specific receptor subtypes), the consequences of these neuromodulatory influences on higher-order network computation are only superficially understood. Through simulations, we show that distinct neuromodulators differentially regulate 5 -HT neuron population coding. For instance, the synapse-specific modulation of glutamatergic drive onto 5-HT and GABA neurons in the DRN by endocannabinoid signalling (Geddes et al., 2016) reduces the overall gain of 5-HT output while maintaining the core preferential encoding of the onset of inputs and maintaining divisive gain modulation. This endocannabinoid normalizing function stands in stark contrast to the fundamental change in the nature of computation triggered by either direct modulation of $I_{A}$ or resting membrane potential (for instance by noradrenergic input; Aghajanian, 1985) that ultimately conditionally tunes the ability of 5-HT neuron populations to encode the derivative of their inputs (Fig. 7F, G, H). These results therefore indicate that different neuromodulators may regulate with remarkable specificity the nature of the computational operation performed by the DRN.

\section{5-HT neurons have strong moving threshold}

Action potential firing in 5-HT neurons leads to a substantial increase in spike threshold (Fig. 4). This strong moving threshold confers adapting properties that compound those provided by the spike-triggered adaptation currents without de-inactivating $I_{A}$. In sensory systems, such spike-triggered adaptation is a ubiquitous property that optimizes neural coding by filtering out temporally redundant information (Brenner et al. 2000; Barlow 2001; Ulanovsky et al. 2003; Kohn 2007). Since redundant information exists at multiple time scales, the optimal filters for redundancy reduction are temporally scale-free, decaying over time according to a power law and implementing a fractional derivative (Lundstrom et al. 2008; Pozzorini et al. 2013). Our results suggest that spike-triggered threshold movement approximately follows a power law in 5-HT neurons. Although the relationship between potent spike-triggered adaptation on multiple timescales and signaling input derivatives is well established in sensory systems, the results reported here highlight the fact that even a neuromodulatory system can prominently signal the derivative of its inputs, suggesting further potential role of adaptation in the DRN (Ranade \& Mainen, 2009; Trulson \& Jacobs 1979).

\section{Role of derivative encoding in reinforcement learning}

The role of 5-HT signalling in modulating behavior is increasingly conceptualized through the lens of reinforcement learning (RL) theory. Indeed, 5-HT output has been proposed to loosely encode or modulate every component of classical RL (Sutton and Barto 2018, Dayan and Huys 2009), including a reward signal (Li et al., 2016), state value (Cohen et al., 2015, Luo et al. 2016), bias in state-action value (Miyazaki et al., 2018), temporal discounting factor (Doya 2002, Schweighofer et al., 2008), prediction error (Daw et al., 2002, but see Boureau and Dayan 2011), and learning rate (Matias et al., 2017, Grossman et al., 2020), with varying degrees of experimental support. Temporal derivatives appear in multiple places in RL, including in the computation of prediction errors from a running history of 
reward signals; and the size/proximity of rewards under temporal discounting, which can be decoded from the rate of change of state (or state-action) value signals. It is interesting to note that the DRN receives value-encoding inputs from the mPFC (Bari et al. 2019, Geddes et al. 2016). In principle, these could provide the basis for a reward size and proximity computation used to control behaviour by promoting patience for impending rewards, a function previously ascribed to 5-HT (Miyazaki et al., 2014, Miyazaki et al., 2018). More recent work has also proposed that 5-HT encodes a learning rate modulation based on unexpected uncertainty, defined as a smoothed temporal derivative of reward prediction error (Grossman et al. 2020). The RPE required by this theory could plausibly be carried by the DRN's copious excitatory ventral tegmental area input (Weissbourd et al. 2014; Aman et al., 2007). Altogether, this work highlights a fundamental computational operation-a temporal derivative-performed by the DRN which arises from the characteristically-strong adaptation mechanisms of 5-HT neurons, raising important questions on the role of the DRN in reward learning and behavioural control. 


\section{Acknowledgements}

This work was carried out on the unceded and unsurrendered land of the Algonquin Anishinaabe people. We thank Dr. Simon Chen for providing SOM-Cre::Rosa-TdTomato mice and Liwen Cai and David Lemelin for technical assistance with mouse lines. We would also like to thank Sébastien Maillé for contributions to pilot-testing of the GIF model in 5-HT neurons and all other members of the J.-C.B. and R.N. labs for helpful discussions. E.H. is thankful to have received graduate scholarships from the Canadian Institutes of Health Research and the Natural Sciences and Engineering Research Council of Canada. This work was supported by grants from the Canadian Institutes of Health Research, the Natural Sciences and Engineering Research Council of Canada, the Canada Foundation for Innovation, Brain Canada (Canadian Neurophotonic Platform), and the Krembil Foundation.

\section{Author Contributions}

Conceptualization: E.H., A.P., M.L., A.L., R.N., and J.-C.B. Design of experiments: E.H., M.L., and A.P. Data collection: E.H., M.L., J.-F.B., L.C.-B., D.C., and C.S. Data analysis, design of aGIF model, design of population models, model fitting, and simulations: E.H. Writing of first draft: E.H. Writing of subsequent drafts: E.H., R.N., and J.-C.B. Review and editing: E.H., R.N., J.-C.B., and A.L. Supervision: R.N. and J.-C.B. Funding acquisition: R.N. and J.-C.B. 


\section{Supplementary Figures}

A

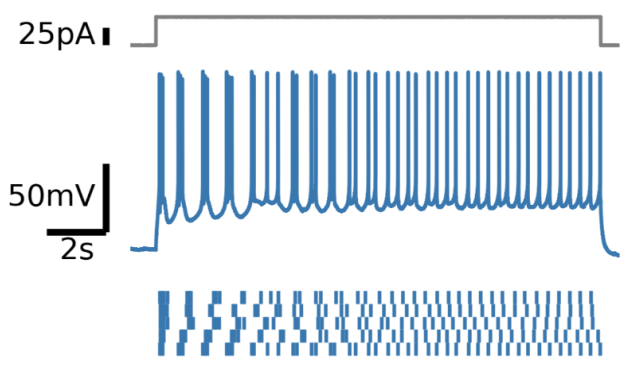

C

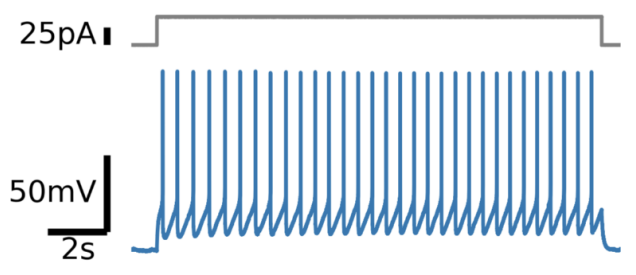

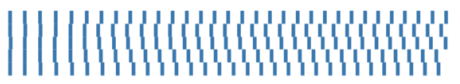

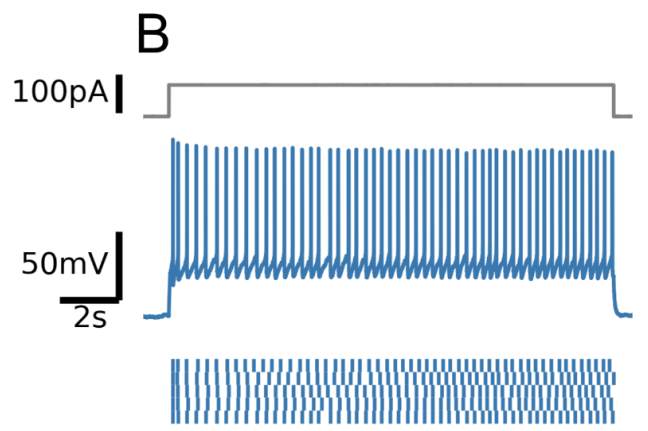

D
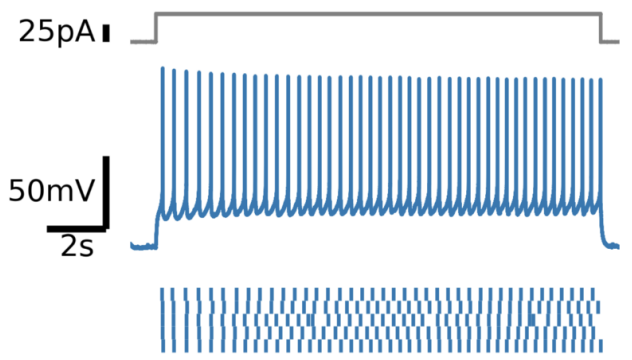

Figure 1-Figure supplement 1 Firing patterns of four positively-identified 5-HT neurons. Note intermittent bursting in the first cell. 

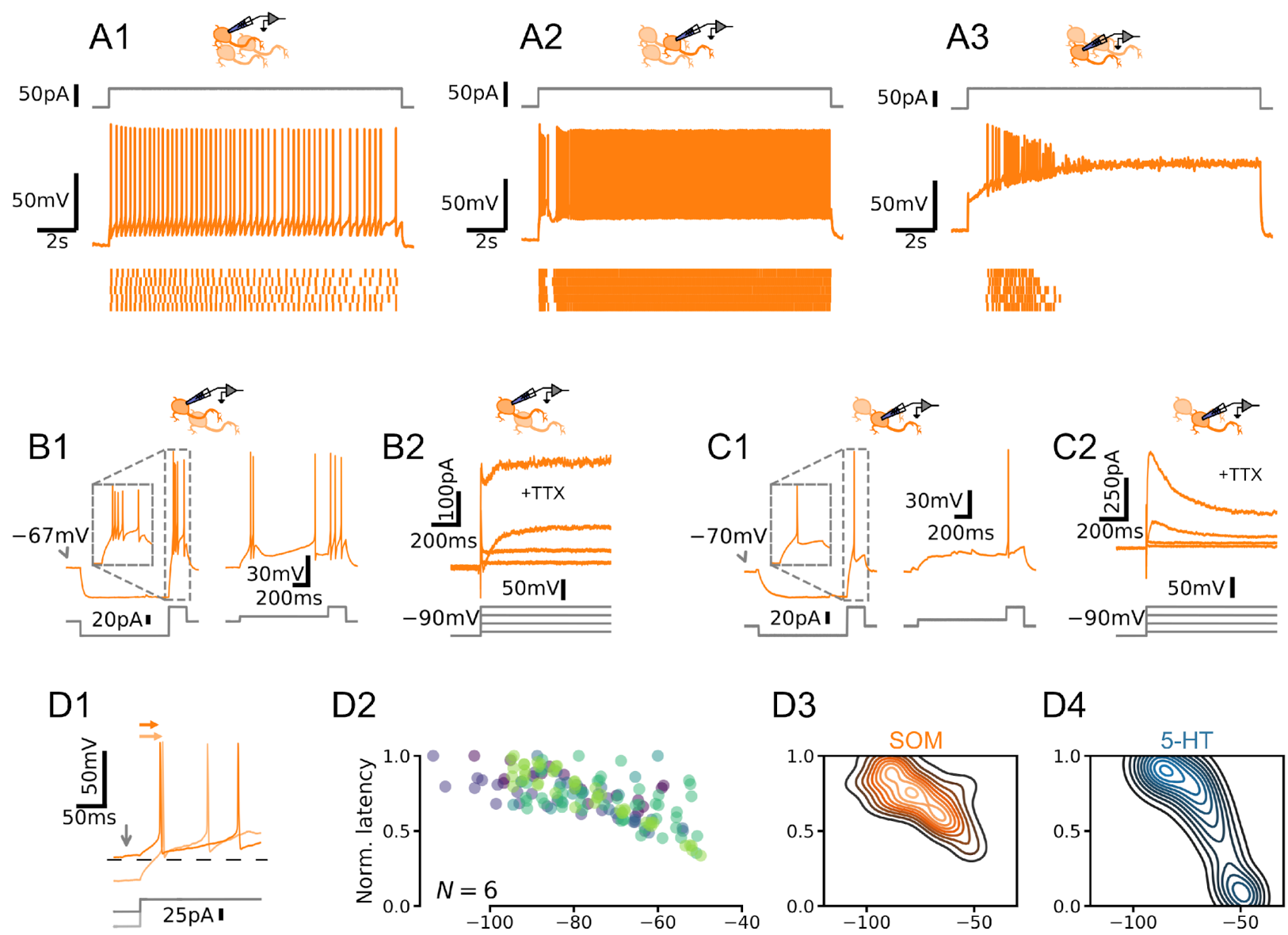

D2
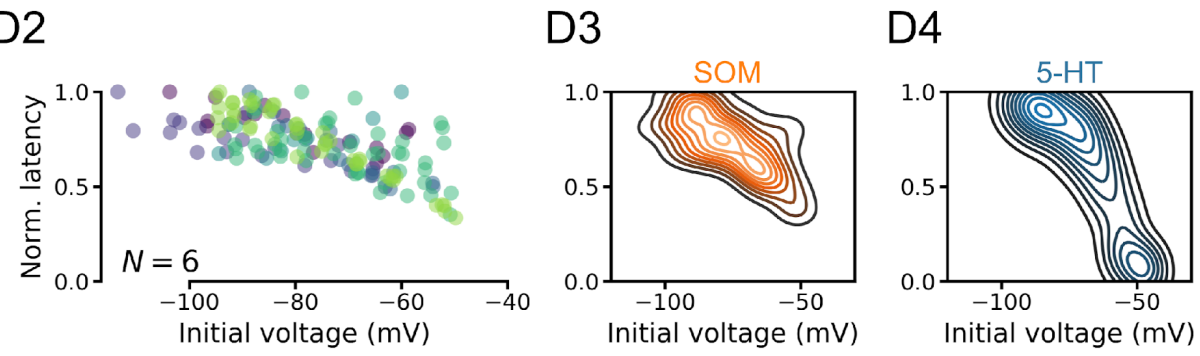

Figure 1-Figure supplement 2 DRN SOM neurons are not homogenous. (A) Qualitatively different firing patterns in three SOM neurons. (B) A burst-firing SOM neuron with a transient depolarizing current. (C) A SOM neuron that does not fire bursts with a pronounced hyperpolarizing current. (D) Heterogenous relationships between voltage history and spike timing across cells. Experiment is the same as in Fig. 2. (D1) Representative experiment. Arrows indicate spike latency and initial voltage. (D2) Normalized spike latency for $N=6 \mathrm{SOM}$ neurons. (D3) Gaussian kernel density estimate of data shown in D2. (D4) Gaussian kernel density estimate of data shown in Fig. $2 F$ for comparison. 


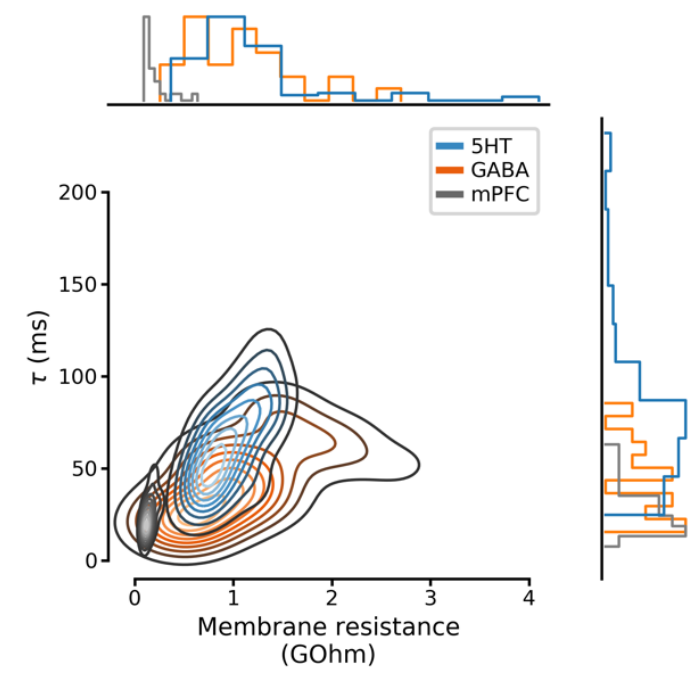

Figure 1-Figure supplement 3 Full distributions of membrane parameters listed in Table 1. Curves on the main set of axes are Gaussian kernel density estimates.

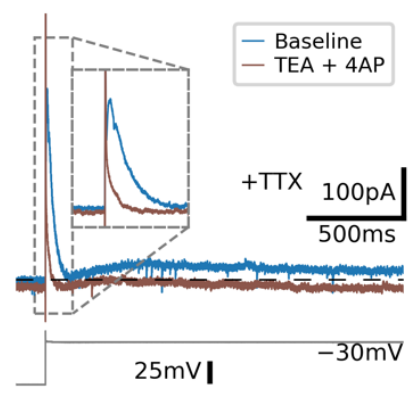

Figure 1-Figure supplement 4 Transient outward current found in 5-HT cells $\left(I_{A}\right)$ is sensitive to potassium channel blockers. 

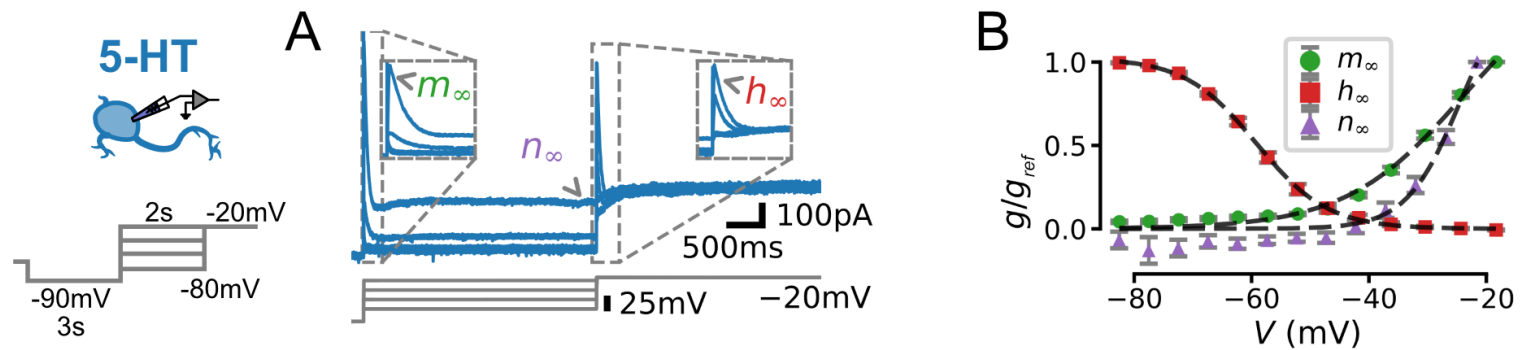

Figure 2-Figure supplement 1 Characterization of voltage-dependence of $I_{A}$ in 5-HT neurons. (A) Experimental protocol. Voltage command designed to reveal voltage-dependent activation and inactivation of $I_{A}($ left $)$ and representative leak-subtracted and capacitance-corrected current trace (blue, top right) aligned to voltage command (gray, bottom right). Parts of the voltage trace used to fit the activation $\left(m_{\infty}\right)$, inactivation $\left(h_{\infty}\right)$, and non-inactivating ( $n_{\infty}$ ) gates (see Methods) are indicated. (B) Gating curves extracted from $N=13$ neurons. $g_{\text {ref }}$ is the maximal conductance observed for each cell (i.e., the conductance at $V=-20 \mathrm{mV}$ for $m_{\infty}$ and $n_{\infty^{\prime}}$ and at $-80 \mathrm{mV}$ for $h_{\infty}$ ). Dashed lines indicate boltzmann functions with parameters given in Table 2. 

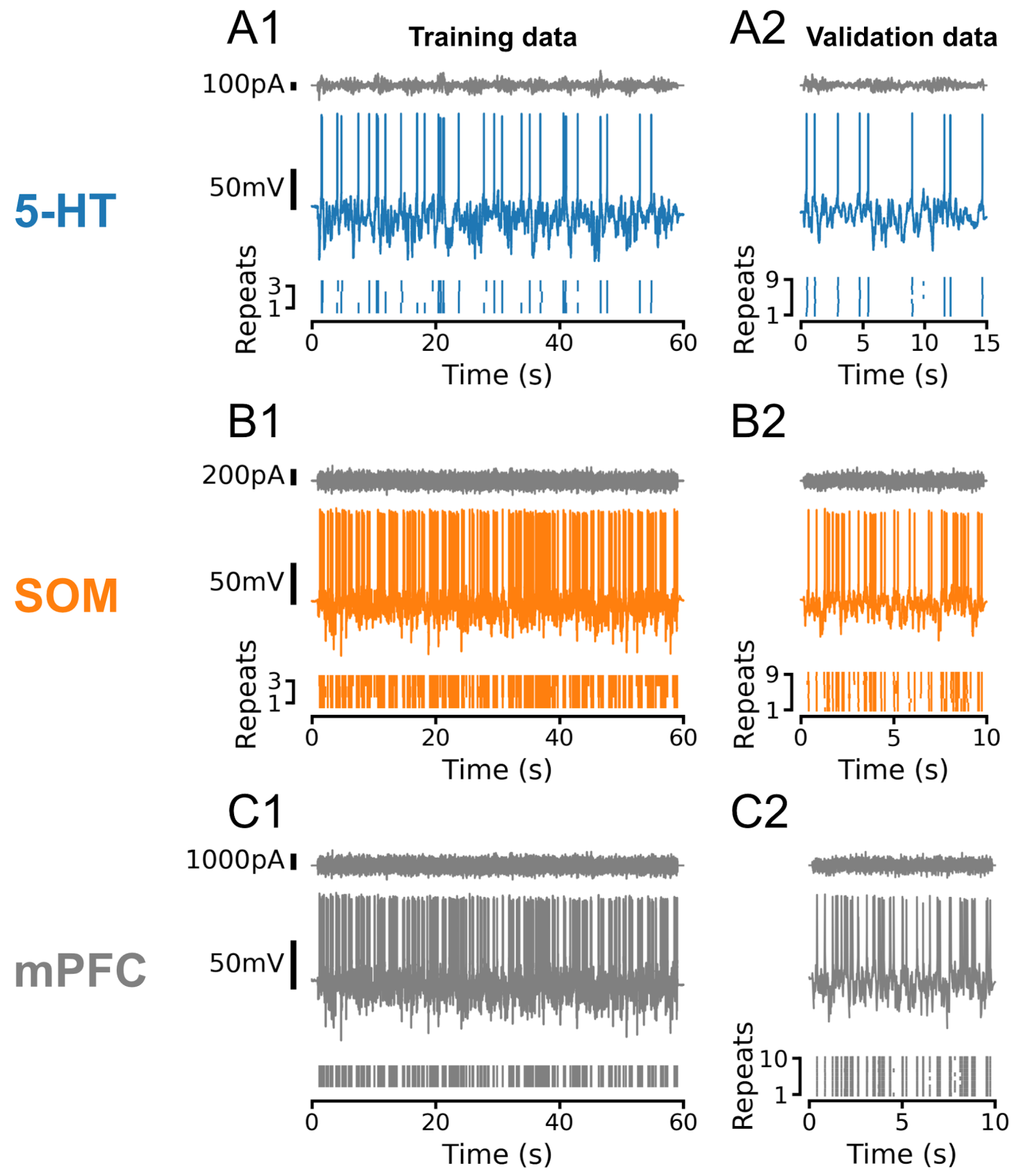

Figure 3-Figure supplement 1 Representative training and validation sets for all cell types. In all cases, training and validation sets consisted of frozen Ornstein-Uhlenbeck $(\mathrm{OU})$ noise with a sinusoidally-modulated variance. Noise was rescaled to evoke subthreshold fluctuations of approximately equal amplitude across individual neurons and cell types. (A) Representative dataset from a 5-HT neuron using OU noise with a time constant $\tau=50 \mathrm{~ms}$ and variance modulations with a period of $5 \mathrm{~s} .(B, C)$ Representative datasets from SOM and mPFC neurons using OU noise with a time constant of $\tau=3$ ms and variance modulations with a period of 100 $m s$. Note that the number and duration of sweeps in the training and test sets varied between cell types, as shown. In 5-HT and SOM neurons, training and validation sweeps were 
interleaved such that each training sweep would be followed by three of the nine validation sweeps. In all cases, active electrode compensation (see Pozzorini et al., 2015) was applied based on a single $10 \mathrm{~s}$ sweep of frozen OU noise with $\tau=3 \mathrm{~ms}$ before fitting and validating GIF models.
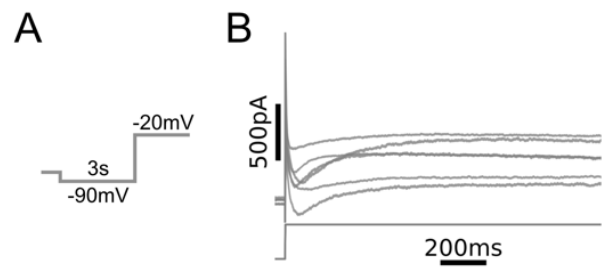

Figure 3-Figure supplement 2 Whole cell currents observed in mPFC neurons. (A) Voltage clamp protocol. Same as in Fig. 1C. (B) Leak-subtracted whole-cell currents from N =6 L5 mPFC pyramidal neurons (top) and voltage command (bottom). Recordings were carried out in the presence of bath-applied tetrodotoxin (TTX).

A Membrane filter

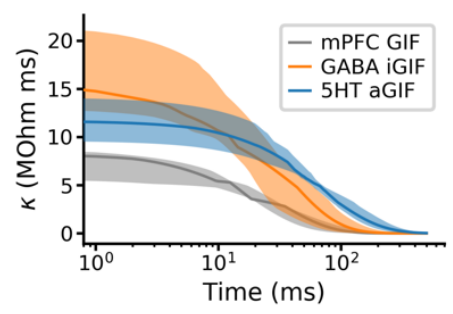

B Spike threshold

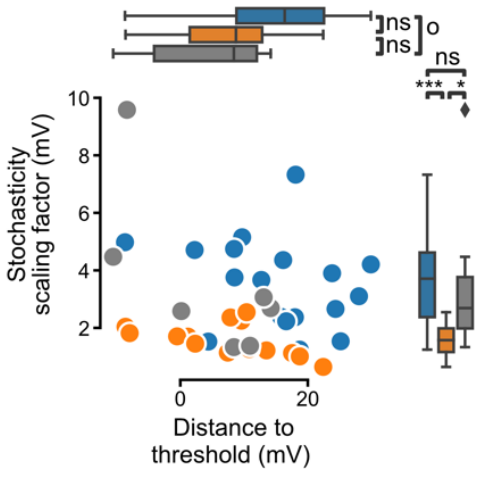

Figure 4-Figure supplement 1 Additional features extracted from single neuron models. (A) Passive membrane filter. Presented as median and interquartile range, as in Fig. $4 C$, D. (B) Spike threshold features: stochasticity scaling factor $(\Delta V$, also known as the threshold width; larger values indicate greater stochasticity) and distance to threshold $\left(V_{T}^{*}-V_{\text {rest }}\right)$. 


\section{A $5-\mathrm{HT}$ in silico}
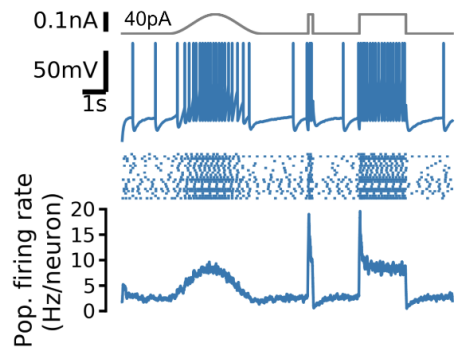

B SOM in sillico

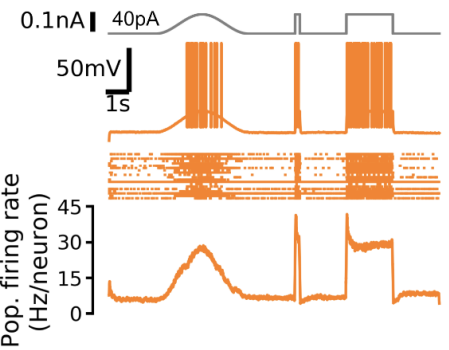

C mPFC in silico

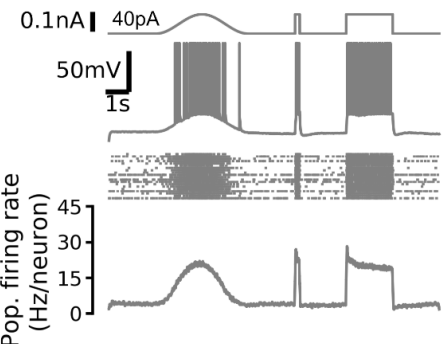

Figure 5-Figure supplement 1 Responses to fast and slow inputs are robust to increases in input baseline. Square step is the same as Fig. 5C but with 40 pA background input. Note strongly-adapting population firing rate in 5-HT neurons. 


\section{A $\quad 5-\mathrm{HT}$ in silico}

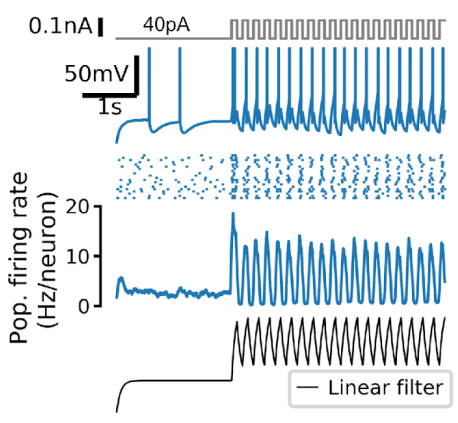

B SOM in silico

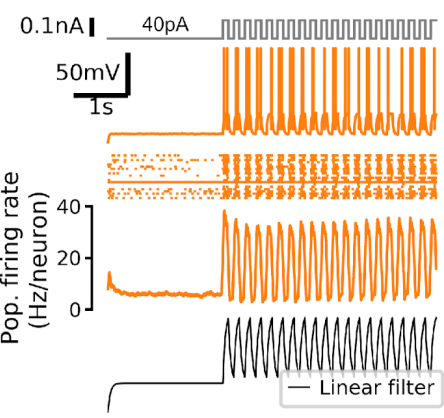

C MPFC in silico

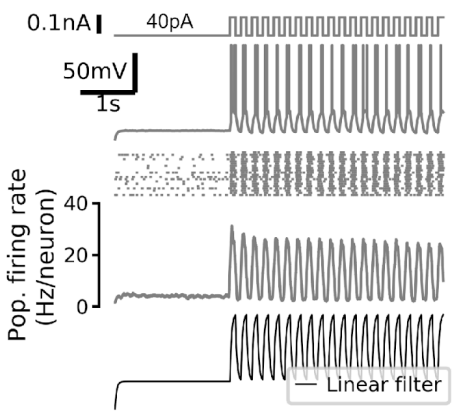

Figure 5-Figure supplement 2 Strong responses of neuron populations to sudden inputs are due to a non-linear filtering effect. A $5 \mathrm{~Hz}$ square wave is applied to each simulated population on top of a constant $40 \mathrm{pA}$ input. Population firing rate shows the mean of $N=20$ independent simulations. Bottom row shows the population firing rate (in arbitrary units) predicted by a linear population coding model (a monoexponential filter with a time constant equal to the mean membrane time constant of the corresponding cell type applied to the input signal). Note that the start of the square wave component of the stimulus is amplified by the simulated neuron populations (second row from bottom) but not by the linear model (bottom row). 

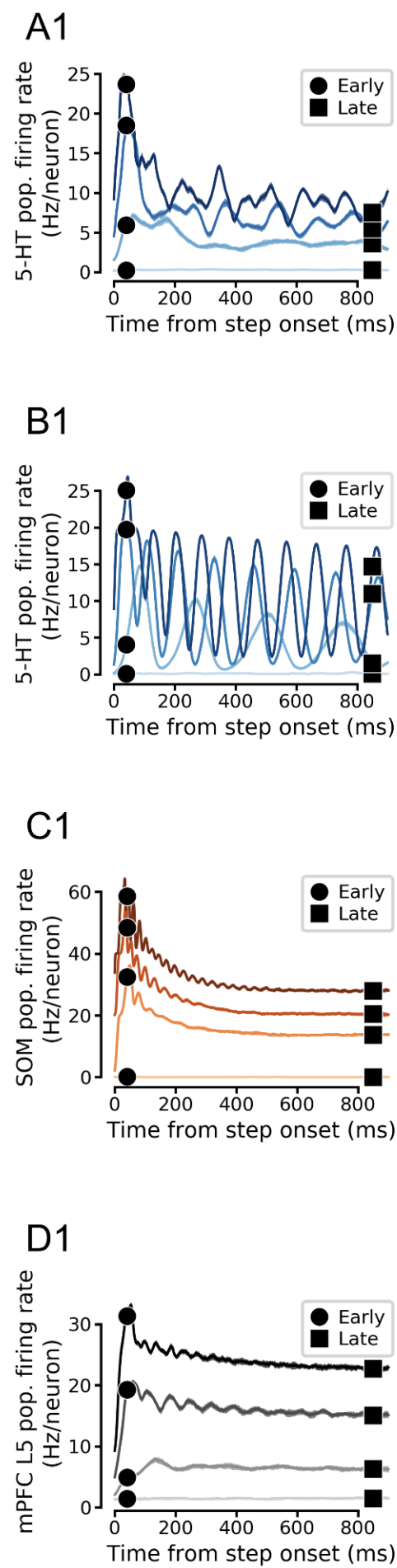

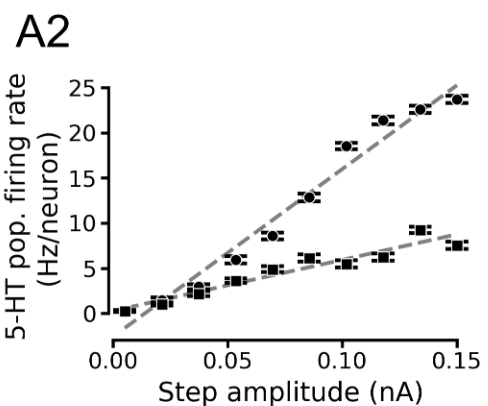

B2

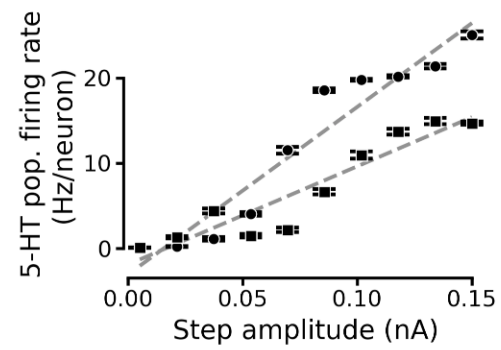

C2

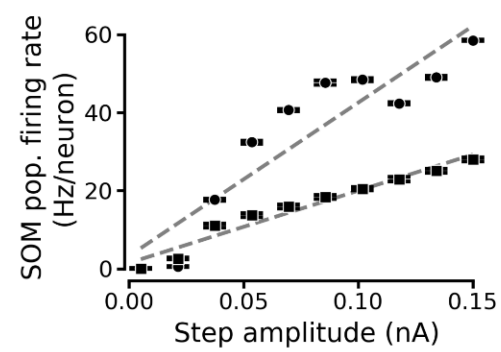

D2

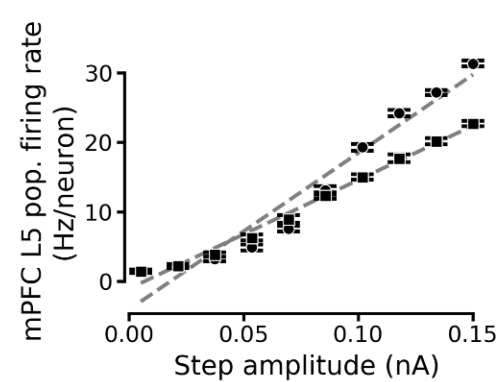

A3

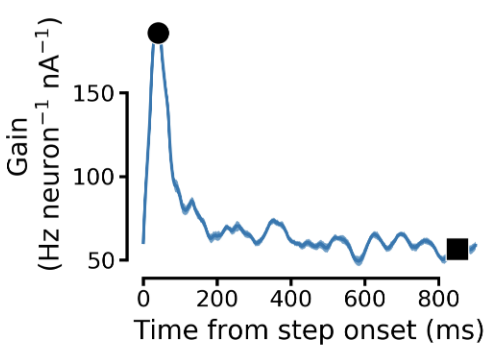

B3

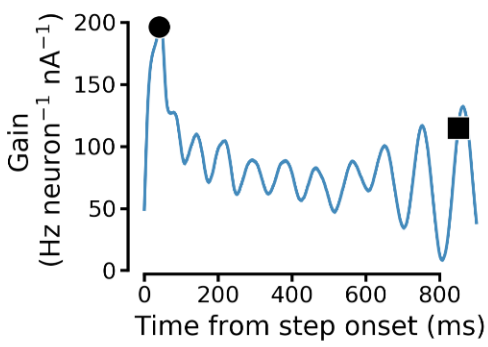

C3

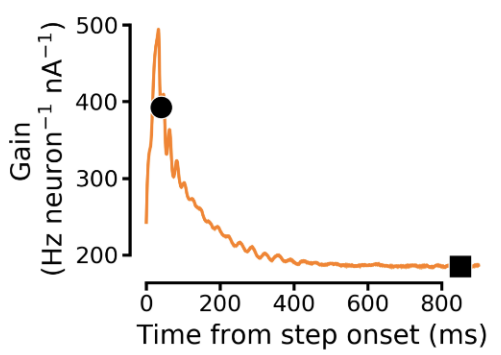

D3

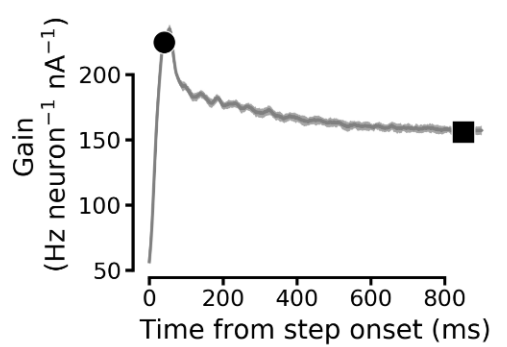

Figure 5-Figure supplement 3 Simulated population input-output functions across cell types. (A) Simulated 5-HT neuron population. (B) Simulated homogenous population of 5-HT neurons. 5-HT neuron models were homogenized by setting each parameter to its median value across all aGIF models fitted to 5-HT neurons. Note oscillations. (C) Simulated SOM neuron population. (D) Simulated mPFC neuron population. Note that the $f$ I curves of 
(heterogenous) 5-HT and SOM neuron populations are approximately linear in the simulated input range ( $A$ and $C$ ). See Fig. 5D for a schematized version of this figure.

A

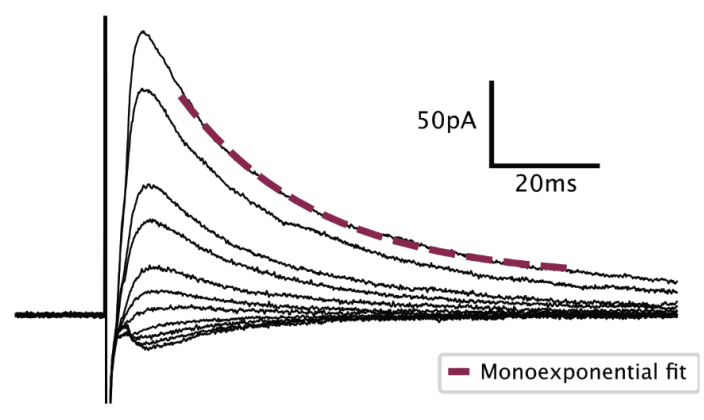

$\mathrm{D}$

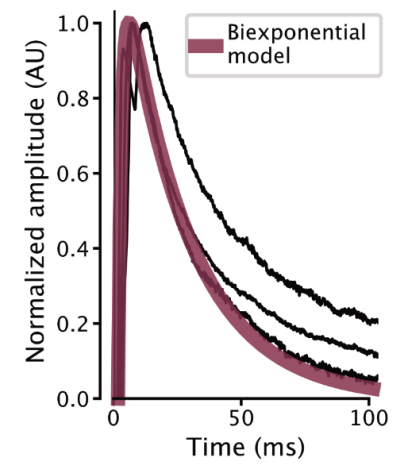

B

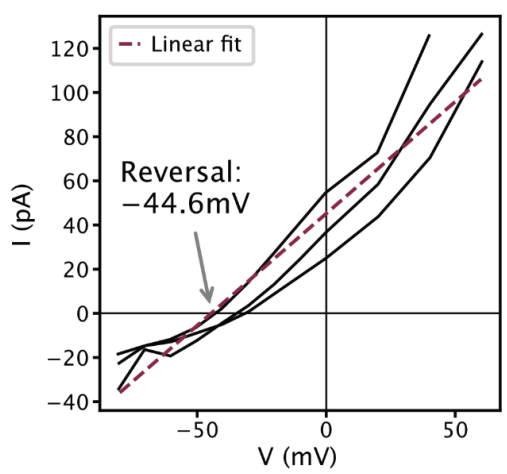

E2

$\mathrm{F}$
C

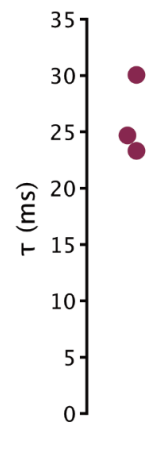

Figure 5-Figure supplement 4 Characterization of GABAergic synapses on 5-HT neurons used to constrain network model. All recordings were carried out in $100 \mu \mathrm{M}$ APV and $5 \mu \mathrm{M}$ NBQX to block glutamatergic synapses. (A) Representative electrically evoked inhibitory post-synaptic currents (eIPSCs) recorded from a 5-HT neuron. Stimulus artefact can be seen just before the start of the synaptic current. (B) Voltage dependence of eIPSC amplitude in $N=$ 3 5-HT neurons. Observed reversal potential is $-44.6 \mathrm{mV}$, consistent with a GABA $A_{A}$ receptor-mediated chloride current under our recording conditions. (C) Fitted decay time constants of eIPSCs from $N=3$ 5-HT neurons held at $60 \mathrm{mV}$. (D) Biexponential GABA synapse model used in DRN network models overlaid on eIPSCs from $N=35$-HT neurons. Model uses $\tau_{\text {rise }}=1.44 \mathrm{~ms}, \tau_{\text {decay }}=26.0 \mathrm{~ms}$, and conductance $0.3 \mathrm{nS}$ (see F). (E) Representative unitary IPSCS recorded in a 5-HT neuron held at $20 \mathrm{mV}$. (F) Distribution of unitary GABA synaptic conductance in 5-HT neurons extracted from unitary IPSCs. Each curve represents $N=308$ 448 unitary IPSCs from a single neuron. IPSC amplitude was converted to units of conductance using $g=\frac{A}{V-V_{\text {rev }}}$, where gis the synaptic conductance, $A$ is the IPSC amplitude, $V=20 \mathrm{mV}$ is the holding potential, and $V_{\text {rev }}=-44.6 \mathrm{mV}$ is the reversal potential of the synaptic current (see B). 


\section{Bibliography}

Abrams, Jolane K., et al. "Anatomic and Functional Topography of the Dorsal Raphe Nucleus." Annals of the New York Academy of Sciences, vol. 1018, no. 1, 2004, pp. 46-57., doi:10.1196/annals.1296.005.

Aman, Teresa K., Roh-Yu Shen, and Samir Haj-Dahmane. "D2-like dopamine receptors depolarize dorsal raphe serotonin neurons through the activation of nonselective cationic conductance." Journal of Pharmacology and Experimental Therapeutics 320, no. 1 (2007): 376-385.

Andrade, Rodrigo. "Serotonergic regulation of neuronal excitability in the prefrontal cortex." Neuropharmacology 61, no. 3 (2011): 382-386.

Aghajanian, G. K. "Modulation of a transient outward current in serotonergic neurones by $\alpha 1$-adrenoceptors." Nature 315.6019 (1985): 501-503.

Aghajanian, G. K., and C. P. Vandermaelen. "Intracellular recordings from serotonergic dorsal raphe neurons: pacemaker potentials and the effects of LSD." Brain research 238.2 (1982): 463-469.

Amo, Ryunosuke, et al. "A Gradual Backward Shift of Dopamine Responses during Associative Learning." 2020, doi:10.1101/2020.10.04.325324.

Amo, Ryunosuke, et al. "The habenulo-raphe serotonergic circuit encodes an aversive expectation value essential for adaptive active avoidance of danger." Neuron 84.5 (2014): 1034-1048.

Baraban, Jay M., and G. K. Aghajanian. "Noradrenergic innervation of serotonergic neurons in the dorsal raphe: demonstration by electron microscopic autoradiography." Brain research 204.1 (1981): 1-11.

Bari, Bilal A., et al. "Stable representations of decision variables for flexible behavior." Neuron 103.5 (2019): 922-933.

Barlow, Horace. "The Exploitation of Regularities in the Environment by the Brain." Behavioral and Brain Sciences, vol. 24, no. 4, 2001, pp. 602-607., doi:10.1017/s0140525x01000024.

Béique, Jean-Claude, Brian Campbell, Paul Perring, Mark W. Hamblin, Paul Walker, Ljiljana Mladenovic, and Rodrigo Andrade. "Serotonergic regulation of membrane potential in developing rat prefrontal cortex: coordinated expression of 5-hydroxytryptamine (5-HT) 1A, 5-HT2A, and 5-HT7 receptors." Journal of Neuroscience 24, no. 20 (2004): 4807-4817.

Béïque, Jean-Claude, Da-Ting Lin, Myoung-Goo Kang, Hiro Aizawa, Kogo Takamiya, and Richard L. Huganir. "Synapse-specific regulation of AMPA receptor function by PSD-95." Proceedings of the National Academy of Sciences 103, no. 51 (2006): 19535-19540.

Béique, Jean-Claude, et al. "Mechanism of the 5-hydroxytryptamine 2A receptor-mediated facilitation of synaptic activity in prefrontal cortex." Proceedings of the National Academy of Sciences 104.23 (2007): 9870-9875.

Benda, Jan, and Andreas V. M. Herz. "A Universal Model for Spike-Frequency Adaptation." Neural Computation, vol. 15, no. 11, 2003, pp. 2523-2564., doi:10.1162/089976603322385063.

Billeh, Yazan N., et al. "Systematic Integration of Structural and Functional Data into Multi-Scale Models of Mouse Primary Visual Cortex." Neuron, vol. 106, no. 3, 2020, doi:10.1016/j.neuron.2020.01.040.

Boureau, Y-Lan, and Peter Dayan. "Opponency revisited: competition and cooperation between dopamine and serotonin." Neuropsychopharmacology 36.1 (2011): 74-97.

Brenner, Naama, William Bialek, and Rob de Ruyter Van Steveninck. "Adaptive rescaling maximizes information transmission." Neuron 26.3 (2000): 695-702.

Calizo, Lyngine H., et al. "Raphe Serotonin Neurons Are Not Homogenous: Electrophysiological, Morphological and Neurochemical Evidence." Neuropharmacology, vol. 61, no. 3, 2011, pp. 524-543., doi:10.1016/j.neuropharm.2011.04.008.

Chance, Frances S, and L F Abbott. "Divisive Inhibition in Recurrent Networks." Network: Computation in Neural Systems, vol. 11, no. 2, 2000, pp. 119-129., doi:10.1088/0954-898x_11_2_301.

Cipriani, Andrea, et al. "Comparative efficacy and acceptability of 21 antidepressant drugs for the acute treatment of adults with major depressive disorder: a systematic review and network meta-analysis." Focus 16.4 (2018): 420-429.

Cohen, Jeremiah Y, et al. "Serotonergic Neurons Signal Reward and Punishment on Multiple Timescales." ELife, 
vol. 4, 2015, doi:10.7554/elife.06346.

Commons, Kathryn G. "Two Major Network Domains in the Dorsal Raphe Nucleus." Journal of Comparative Neurology, vol. 523, no. 10, 2015, pp. 1488-1504., doi:10.1002/cne.23748.

Connor, J. A., and C. F. Stevens. "Prediction of Repetitive Firing Behaviour from Voltage Clamp Data on an Isolated Neurone Soma." The Journal of Physiology, vol. 213, no. 1, 1971, pp. 31-53., doi:10.1113/jphysiol.1971.sp009366.

Connor, J.a., et al. "Neural Repetitive Firing: Modifications of the Hodgkin-Huxley Axon Suggested by Experimental Results from Crustacean Axons." Biophysical Journal, vol. 18, no. 1, 1977, pp. 81-102., doi:10.1016/s0006-3495(77)85598-7.

Cools, Roshan, Kae Nakamura, and Nathaniel D. Daw. "Serotonin and dopamine: unifying affective, activational, and decision functions." Neuropsychopharmacology 36.1 (2011): 98-113.

Daw, Nathaniel D, et al. "Opponent Interactions between Serotonin and Dopamine." Neural Networks, vol. 15, no. 4-6, 2002, pp. 603-616., doi:10.1016/s0893-6080(02)00052-7.

Dayan, Peter, and Quentin J.m. Huys. "Serotonin in Affective Control." Annual Review of Neuroscience, vol. 32, no. 1, 2009, pp. 95-126., doi:10.1146/annurev.neuro.051508.135607.

Dayan, Peter, and Quentin Huys. "Neurophysiology: Serotonin's many meanings elude simple theories." Elife 4 (2015): e07390.

Deakin, J.f.w. “Serotonin in Panic, Anxiety and Depression." European Neuropsychopharmacology, vol. 1, no. 3, 1991, pp. 358-360., doi:10.1016/0924-977x(91)90566-d.

Delgado, Pedro L. "Serotonin and the Neurobiology of Depression." Archives of General Psychiatry, vol. 51, no. 11, 1994, p. 865., doi:10.1001/archpsyc.1994.03950110025005.

Drion, Guillaume, et al. "Ion Channel Degeneracy Enables Robust and Tunable Neuronal Firing Rates." Proceedings of the National Academy of Sciences, vol. 112, no. 38, 2015, doi:10.1073/pnas.1516400112.

Donaldson, Zoe R., et al. "Genetic approaches for understanding the role of serotonin receptors in mood and behavior." Current opinion in neurobiology 23.3 (2013): 399-406.

Dong, Yan, and Francis J. White. "Dopamine D1-class receptors selectively modulate a slowly inactivating potassium current in rat medial prefrontal cortex pyramidal neurons." Journal of Neuroscience 23.7 (2003): 2686-2695.

Dong, Yan, et al. "Cocaine-induced plasticity of intrinsic membrane properties in prefrontal cortex pyramidal neurons: adaptations in potassium currents." Journal of Neuroscience 25.4 (2005): 936-940.

Doya, Kenji. "Metalearning and neuromodulation." Neural networks 15.4-6 (2002): 495-506.

Ermentrout, Bard. "Linearization of F-I Curves by Adaptation." Neural Computation, vol. 10, no. 7, 1998, pp. 1721-1729., doi:10.1162/089976698300017106.

Fava, Maurizio, and Kenneth S Kendler. “Major Depressive Disorder." Neuron, vol. 28, no. 2, 2000, pp. 335-341., doi:10.1016/s0896-6273(00)00112-4.

Ferguson, Katie A., and Jessica A. Cardin. "Mechanisms underlying gain modulation in the cortex." Nature Reviews Neuroscience 21, no. 2 (2020): 80-92.

Fonseca, Madalena S., et al. "Activation of Dorsal Raphe Serotonergic Neurons Promotes Waiting but Is Not Reinforcing." Current Biology, vol. 25, no. 3, 2015, pp. 306-315., doi:10.1016/j.cub.2014.12.002.

Geddes, Sean D., et al. "Target-Specific Modulation of the Descending Prefrontal Cortex Inputs to the Dorsal Raphe Nucleus by Cannabinoids." Proceedings of the National Academy of Sciences, vol. 113, no. 19, 2016, pp. 5429-5434., doi:10.1073/pnas.1522754113.

Gerstner, W., and R. Naud. "How Good Are Neuron Models?" Science, vol. 326, no. 5951, 2009, pp. 379-380., doi:10.1126/science.1181936.

Gerstner, Wulfram, et al. Neuronal Dynamics from Single Neurons to Networks and Models of Cognition. Cambridge University Press, 2014.

Gerstner, Wulfram. "Population Dynamics of Spiking Neurons: Fast Transients, Asynchronous States, and Locking." Neural Computation, vol. 12, no. 1, 2000, pp. 43-89., doi:10.1162/089976600300015899.

Getting, Peter A. "Mechanisms of pattern generation underlying swimming in Tritonia. III. Intrinsic and synaptic mechanisms for delayed excitation." Journal of Neurophysiology 49.4 (1983): 1036-1050.

Gonçalves, Pedro J., et al. "Training Deep Neural Density Estimators to Identify Mechanistic Models of Neural Dynamics." 2019, doi:10.1101/838383.

Grossman, Cooper D., Bilal A. Bari, and Jeremiah Y. Cohen. "Serotonin neurons modulate learning rate through uncertainty." bioRxiv (2020).

Harnett, Mark T., et al. "Potassium Channels Control the Interaction between Active Dendritic Integration 
Compartments in Layer 5 Cortical Pyramidal Neurons." Neuron, vol. 79, no. 3, 2013, pp. 516-529., doi:10.1016/j.neuron.2013.06.005.

Hoffman, Dax A., et al. "K Channel Regulation of Signal Propagation in Dendrites of Hippocampal Pyramidal Neurons." Nature, vol. 387, no. 6636, 1997, pp. 869-875., doi:10.1038/43119.

Huys, Quentin J. M., et al. "Efficient Estimation of Detailed Single-Neuron Models." Journal of Neurophysiology, vol. 96, no. 2, 2006, pp. 872-890., doi:10.1152/jn.00079.2006.

Huys, Quentin JM, and Liam Paninski. "Smoothing of, and parameter estimation from, noisy biophysical recordings." PLoS Comput Biol 5.5 (2009): e1000379.

Knight, Bruce W. "Dynamics of Encoding in a Population of Neurons." Journal of General Physiology, vol. 59, no. 6, 1972, pp. 734-766., doi:10.1085/jgp.59.6.734.

Kohn, Adam. "Visual Adaptation: Physiology, Mechanisms, and Functional Benefits." Journal of Neurophysiology, vol. 97, no. 5, 2007, pp. 3155-3164., doi:10.1152/jn.00086.2007.

$\mathrm{Li}, \mathrm{Yi}$, et al. "Serotonin Neurons in the Dorsal Raphe Nucleus Encode Reward Signals." Nature Communications, vol. 7, no. 1, 2016, doi:10.1038/ncomms10503.

Lowry, Christopher A., et al. "Modulation of Anxiety Circuits by Serotonergic Systems." Stress, vol. 8, no. 4, 2005, pp. 233-246., doi:10.1080/10253890500492787.

Lundstrom, Brian N, et al. "Fractional Differentiation by Neocortical Pyramidal Neurons." Nature Neuroscience, vol. 11, no. 11, 2008, pp. 1335-1342., doi:10.1038/nn.2212.

Luo, Minmin, et al. "Do Dorsal Raphe 5-HT Neurons Encode 'Beneficialness'?" Neurobiology of Learning and Memory, vol. 135, 2016, pp. 40-49., doi:10.1016/j.nlm.2016.08.008.

Maier, Steven F., and Linda R. Watkins. "Stressor Controllability and Learned Helplessness: The Roles of the Dorsal Raphe Nucleus, Serotonin, and Corticotropin-Releasing Factor." Neuroscience; Biobehavioral Reviews, vol. 29, no. 4-5, 2005, pp. 829-841., doi:10.1016/j.neubiorev.2005.03.021.

Marder, Eve. "Neuromodulation of neuronal circuits: back to the future." Neuron 76, no. 1 (2012): 1-11.

Markram, Henry. "The Blue Brain Project." Nature Reviews Neuroscience, vol. 7, no. 2, 2006, pp. 153-160., doi:10.1038/nrn1848.

Matias, Sara, et al. "Activity patterns of serotonin neurons underlying cognitive flexibility." Elife 6 (2017): e20552.

Matsumoto, Masayuki, and Okihide Hikosaka. "Lateral habenula as a source of negative reward signals in dopamine neurons." Nature 447.7148 (2007): 1111-1115.

McCormick, DAVID A. "Functional properties of a slowly inactivating potassium current in guinea pig dorsal lateral geniculate relay neurons." Journal of Neurophysiology 66.4 (1991): 1176-1189.

Mejias, J. F., and A. Longtin. "Optimal Heterogeneity for Coding in Spiking Neural Networks." Physical Review Letters, vol. 108, no. 22, 2012, doi:10.1103/physrevlett.108.228102.

Mejias, Jorge F., and André Longtin. "Differential Effects of Excitatory and Inhibitory Heterogeneity on the Gain and Asynchronous State of Sparse Cortical Networks." Frontiers in Computational Neuroscience, vol. 8, 2014, doi:10.3389/fncom.2014.00107.

Mejias, Jorge F., et al. "Subtractive, Divisive and Non-Monotonic Gain Control in Feedforward Nets Linearized by Noise and Delays." Frontiers in Computational Neuroscience, vol. 8, 2014, doi:10.3389/fncom.2014.00019.

Mensi, Skander, et al. "Enhanced Sensitivity to Rapid Input Fluctuations by Nonlinear Threshold Dynamics in Neocortical Pyramidal Neurons." PLOS Computational Biology, vol. 12, no. 2, 2016, doi:10.1371/journal.pcbi.1004761.

Mensi, Skander, et al. "Parameter Extraction and Classification of Three Cortical Neuron Types Reveals Two Distinct Adaptation Mechanisms." Journal of Neurophysiology, vol. 107, no. 6, 2012, pp. 1756-1775., doi:10.1152/jn.00408.2011.

Miyazaki, Kayoko W., et al. "Optogenetic Activation of Dorsal Raphe Serotonin Neurons Enhances Patience for Future Rewards." Current Biology, vol. 24, no. 17, 2014, pp. 2033-2040., doi:10.1016/j.cub.2014.07.041.

Miyazaki, Katsuhiko, et al. "Reward probability and timing uncertainty alter the effect of dorsal raphe serotonin neurons on patience." Nature communications 9.1 (2018): 1-11.

Muzerelle, Aude, et al. "Conditional Anterograde Tracing Reveals Distinct Targeting of Individual Serotonin Cell Groups (B5-B9) to the Forebrain and Brainstem." Brain Structure and Function, vol. 221, no. 1, 2014, pp. 535-561., doi:10.1007/s00429-014-0924-4.

Nakamura, K., et al. "Reward-Dependent Modulation of Neuronal Activity in the Primate Dorsal Raphe 
Nucleus." Journal of Neuroscience, vol. 28, no. 20, 2008, pp. 5331-5343., doi:10.1523/jneurosci.0021-08.2008.

Naud, Richard, and Wulfram Gerstner. "Coding and Decoding with Adapting Neurons: A Population Approach to the Peri-Stimulus Time Histogram." PLoS Computational Biology, vol. 8, no. 10, 2012, doi:10.1371/journal.pcbi.1002711.

Naud, Richard, et al. "Improved Similarity Measures for Small Sets of Spike Trains." Neural Computation, vol. 23, no. 12, 2011, pp. 3016-3069., doi:10.1162/neco_a_00208.

Ogawa, Sachie K., et al. "Organization of Monosynaptic Inputs to the Serotonin and Dopamine Neuromodulatory Systems." Cell Reports, vol. 8, no. 4, 2014, pp. 1105-1118., doi:10.1016/j.celrep.2014.06.042.

Okaty, Benjamin W., et al. "Embracing Diversity in the 5-HT Neuronal System." Nature Reviews Neuroscience, vol. 20, no. 7, July 2019, pp. 397-424, doi:10.1038/s41583-019-0151-3.

Paninski, Liam, et al. "Comparing Integrate-and-Fire Models Estimated Using Intracellular and Extracellular Data." Neurocomputing, vol. 65-66, 2005, pp. 379-385., doi:10.1016/j.neucom.2004.10.032.

Payeur, Alexandre, et al. "Classes of Dendritic Information Processing." Current Opinion in Neurobiology, vol. 58, 2019, pp. 78-85., doi:10.1016/j.conb.2019.07.006.

Pillow, Jonathan W., et al. "Spatio-Temporal Correlations and Visual Signalling in a Complete Neuronal Population." Nature, vol. 454, no. 7207, 2008, pp. 995-999., doi:10.1038/nature07140.

Pollak Dorocic, Iskra, et al. "A Whole-Brain Atlas of Inputs to Serotonergic Neurons of the Dorsal and Median Raphe Nuclei." Neuron, vol. 83, no. 3, 2014, pp. 663-678., doi:10.1016/j.neuron.2014.07.002.

Pozzorini, Christian, et al. "Automated High-Throughput Characterization of Single Neurons by Means of Simplified Spiking Models." PLOS Computational Biology, vol. 11, no. 6, 2015, doi:10.1371/journal.pcbi.1004275.

Pozzorini, Christian, et al. "Temporal Whitening by Power-Law Adaptation in Neocortical Neurons." Nature Neuroscience, vol. 16, no. 7, 2013, pp. 942-948., doi:10.1038/nn.3431.

Prinz, Astrid A, et al. "Similar Network Activity from Disparate Circuit Parameters." Nature Neuroscience, vol. 7, no. 12, 2004, pp. 1345-1352., doi:10.1038/nn1352.

Ranade, Sachin P., and Zachary F. Mainen. "Transient Firing of Dorsal Raphe Neurons Encodes Diverse and Specific Sensory, Motor, and Reward Events." Journal of Neurophysiology, vol. 102, no. 5, 2009, pp. 3026-3037., doi:10.1152/jn.00507.2009.

Ren, Jing, et al. "Anatomically Defined and Functionally Distinct Dorsal Raphe Serotonin Sub-Systems." Cell, vol. 175, no. 2, 2018, doi:10.1016/j.cell.2018.07.043.

Savitz, Jonathan, Irwin Lucki, and Wayne C. Drevets. "5-HT1A receptor function in major depressive disorder." Progress in neurobiology 88.1 (2009): 17-31.

Schweighofer, Nicolas, et al. "Low-serotonin levels increase delayed reward discounting in humans." Journal of Neuroscience 28.17 (2008): 4528-4532.

Schweimer, J.v., and M.a. Ungless. "Phasic Responses in Dorsal Raphe Serotonin Neurons to Noxious Stimuli." Neuroscience, vol. 171, no. 4, 2010, pp. 1209-1215., doi:10.1016/j.neuroscience.2010.09.058.

Segal, Menahem. "A potent transient outward current regulates excitability of dorsal raphe neurons." Brain research 359.1-2 (1985): 347-350.

Soubrié, Philippe. "Reconciling the role of central serotonin neurons in human and animal behavior." Behavioral and Brain Sciences 9.2 (1986): 319-335.

Storm, J F. "An after-Hyperpolarization of Medium Duration in Rat Hippocampal Pyramidal Cells." The Journal of Physiology, vol. 409, no. 1, 1989, pp. 171-190., doi:10.1113/jphysiol.1989.sp017491.

Sutton, Richard S., and Andrew Barto. Reinforcement Learning: an Introduction. The MIT Press, 2018.

Teeter, Corinne, et al. "Generalized Leaky Integrate-and-Fire Models Classify Multiple Neuron Types." Nature Communications, vol. 9, no. 1, 2018, doi:10.1038/s41467-017-02717-4.

Tops, Mattie, et al. "Serotonin: Modulator of a Drive to Withdraw." Brain and Cognition, vol. 71, no. 3, 2009, pp. 427-436., doi:10.1016/j.bandc.2009.03.009.

Trulson, Michael E., and Barry L. Jacobs. "Raphe Unit Activity in Freely Moving Cats: Correlation with Level of Behavioral Arousal." Brain Research, vol. 163, no. 1, 1979, pp. 135-150., doi:10.1016/0006-8993(79)90157-4.

Tsuda, Ben, et al. "Neuromodulators enable overlapping synaptic memory regimes and nonlinear transition dynamics in recurrent neural networks." bioRxiv, 2021, doi:10.1101/2021.05.31.446462.

Tuckwell, Henry C., and Nicholas J. Penington. "Computational Modeling of Spike Generation in Serotonergic 
Neurons of the Dorsal Raphe Nucleus." Progress in Neurobiology, vol. 118, 2014, pp. 59-101., doi:10.1016/j.pneurobio.2014.04.001.

Ujfalussy, Balázs B., et al. "Global and multiplexed dendritic computations under in vivo-like conditions." Neuron 100.3 (2018): 579-592.

Ulanovsky, Nachum, et al. "Processing of Low-Probability Sounds by Cortical Neurons." Nature Neuroscience, vol. 6, no. 4, 2003, pp. 391-398., doi:10.1038/nn1032.

Vandermaelen, C. P., and G. K. Aghajanian. "Electrophysiological and pharmacological characterization of serotonergic dorsal raphe neurons recorded extracellularly and intracellularly in rat brain slices." Brain research 289.1-2 (1983): 109-119.

Varga, Viktor, et al. "Fast synaptic subcortical control of hippocampal circuits." Science 326.5951 (2009): 449-453.

Warden, Melissa R., et al. "A Prefrontal Cortex-Brainstem Neuronal Projection That Controls Response to Behavioural Challenge." Nature, vol. 492, no. 7429, 2012, pp. 428-432., doi:10.1038/nature11617.

Weissbourd, Brandon, et al. "Presynaptic Partners of Dorsal Raphe Serotonergic and GABAergic Neurons." Neuron, vol. 83, no. 3, 2014, pp. 645-662., doi:10.1016/j.neuron.2014.06.024.

Wong-Lin, Kongfatt, et al. "A Spiking Neuronal Network Model of the Dorsal Raphe Nucleus." The 2011 International Joint Conference on Neural Networks, 2011, doi:10.1109/ijcnn.2011.6033414.

Young, Simon N., et al. "Tryptophan Depletion Causes a Rapid Lowering of Mood in Normal Males." Psychopharmacology, vol. 87, no. 2, 1985, pp. 173-177., doi:10.1007/bf00431803.

Zhong, Weixin, et al. "Learning and Stress Shape the Reward Response Patterns of Serotonin Neurons." The Journal of Neuroscience, vol. 37, no. 37, 2017, pp. 8863-8875., doi:10.1523/jneurosci.1181-17.2017.

Zhou, Li, et al. "Organization of Functional Long-Range Circuits Controlling the Activity of Serotonergic Neurons in the Dorsal Raphe Nucleus." Cell Reports, vol. 18, no. 12, 2017, pp. 3018-3032., doi:10.1016/j.celrep.2017.02.077. 